\title{
A Simple and Convenient Synthesis of Isolated-Fused Heterocycles Based on: 2-Imino- $N$-phenyl-2H-chromene-3-carboxamide
}

\author{
Islam H. El Azab*, Fawi M. Abd El Latif \\ Chemistry Department, Faculty of Science, Aswan University, Aswan, Egypt \\ Email: *ihelmy2003@yahoo.com
}

Received June 23, 2012; revised July 26, 2012; accepted August 29, 2012

\begin{abstract}
Starting from 2-imino- $N$-phenyl-2H-chromene-3-carbox-amide, (1) a series of functionalized chromenes were achieved; such as, 2-ethoxy-2,3-dihydro-3-phenylchromeno[2,3- $d$ ]pyrimidin-4-one (2), and 2-hydrazinyl-2,3-dihydro-3-phenylchromeno-[2,3- $d$ ] pyrimidin-4-one (3). Furthermore, reactions of (3) with some of laboratory available compounds gave pyrazoles $(\mathbf{4}-\mathbf{9}, \mathbf{1 2}$, 13a, 13b), tetrazoles (11), 2-(2-benzylidenehydrazinyl)-3-phenyl-3H-chromeno[2,3- $d]$ pyrimidin4(10H)-oneisoxazoles (14), 5-chloro-1-(4-oxo-3-phenyl-4,10-dihydro-3H-chromeno[2,3-d]pyrimidin-2-yl)-3-phenyl-2, 3-dihydro-1H-pyrazole-4-carbonitrile (17), pyrimidines (28a, b), pyridines (29a - 29e, 30, 33a, 33b), benzo[b][1, 4] oxazepin-2- amines (32a, b), 3-chloro-4-(2-imino-2H-chromen-3-yl)-1-phenyl-4-(phenylamino) azetidin-2-one (34a34e) and 2-(2- imino-2H-chromen-3-yl)-3-phenyl-2-(phenyl amino)thiazolidin-4-onee (35a -35e). The structures of these compounds were established by elemental analysis, IR, MS and NMR spectral analysis.
\end{abstract}

Keywords: 2-Imino-2 $H$-chromen-3-yl; Chromeno[2,3- $d$ ]pyrimidin-4-one; $\beta$-Lactam; Thiazolidin-4-ones

\section{Introduction}

Natural and synthetic coumarin derivatives represent, nowadays, an important group of organic compounds that are used as antibiotics [1,2] fungicides [3] anti-inflammatory [4], anticoagulant [5] and antitumor agents [6,7]. Regarding their high fluorescence ability, they are widely used as optical whitening agents, brighteners, laser dyes and also as fluorescent probes [8] in biology and medicine [9]. Also, The $4 \mathrm{H}$-chromene derivatives ethyl 4-((ethoxycarbonyl) (cyano) methyl)-2-amino-6-bromo- $4 H$-chromene3-carboxylate (HA 14-1) has demonstrated promising antifungal activities [10], antiviral agent [11], antiproliferation agent [12]. Due to the unique biological and pharmacological activity, chromene derivatives have attracted considerable attention thus; different processes for the synthesis of chromenes have been reported during the past few years. The importance of the chromone nucleus is evidenced by the continued appearance of new and improved methods for their synthesis, despite the several existing methods for the synthesis of chromene derivatives [13-20], there still is demand for general synthetic strategies which can efficiently provide variously substituted chromene systems.

\footnotetext{
"Corresponding author.
}

\section{Experimental}

\subsection{Instruments}

All melting points are measured using Galenkanp melting point apparatus and are uncorrected. Elemental analyses were carried out at the Microanalytical Center of Cairo University. IR ( $\mathrm{KBr}$ pellets $v=\mathrm{cm}^{-1}$ ) spectra were determined in 1650 FT-IR instrument (Cairo University), 1H-NMR spectra ( $\delta=\mathrm{ppm})$ were accomplished using 300 MHz NMR Spectrometer and mass spectroscopy were recorded on GCMS-QP-1000 EX spectrometer (Cairo University).

\subsection{Material and Reagents}

Hydrazine hydrate, phenyhydrazine, benzaldehyde and its substituted derivatives, aniline and its substituted derivatives, thioglycolic acid, acetylacetone, phosoryl chloride, ethyl acetoacetate and chloroacetyl chloride were purchased from Alderich Chemical Co.

Triethylamine, thiourea, urea, o-phenylenediamine, oaminothiophenol and 2-cyanomethy benzimidazole were purchased from British Drug Houses (BDH).

Acetophenone, malononitrile, 2-chloro acetamide, piperidine, ethoxymethylene-malononitrile, sodium azide and 
5-amino-(1H)-1,2,4-triazole were purchased from Merck Co., Germany.

\subsection{Solvents}

Dimethyformamide, benzene, pyridine, ethanol and acetone were purchased from El-Nasr Pharmaceutical and Chemical Co. (ADWIC), Egypt.

\subsection{Organic Preparations}

Preparation of 2-ethoxy-2, 3-dihydro-3-phenylchromeno[2,3- $d$ ]pyrimidin-4-one (2). A mixture of 1 (2.64 g, $0.01 \mathrm{~mol})$ and triethoxymethane $(1.48 \mathrm{~mL}, 0.05 \mathrm{~mol})$ in 20 $\mathrm{mL}$ of dimethylformamide was refluxed $8 \mathrm{~h}$. Then the reaction mixture was poured into $150 \mathrm{~mL}$ of crushed ice then the resultant solid was collected by filtration to provide $2(1.5 \mathrm{~g}, 60 \%)$ as a pale yellow solid; m.p. $285^{\circ} \mathrm{C}$ $287^{\circ} \mathrm{C} .{ }^{1} \mathrm{HNMR}\left(\mathrm{CDCl}_{3}\right): \delta 1.2\left(t, 3 \mathrm{H}, \mathrm{CH}_{3}\right), 3.9(q, 2 \mathrm{H}$, $\left.\mathrm{CH}_{2}\right)$ and $6.7-7.9(m, 11 \mathrm{H}, \mathrm{Ar}-\mathrm{H})$. MS: $m / z 320\left([\mathrm{M}]^{+}\right.$, $65 \%$ ). Calcd for $\mathrm{C}_{19} \mathrm{H}_{16} \mathrm{~N}_{2} \mathrm{O}_{3}$ (320.34). Calcd: C 71.24, $\mathrm{H}$ 5.03, N 8.74 \%. Found: C 70.14, H 5.01, N 7.88\%.

Preparation of 2-hydrazinyl-2, 3-dihydro-3-phenyl chromeno[2,3- $d$ ]pyrim-idin-4-one (3). A mixture of 2 $(3.20 \mathrm{~g}, 0.01 \mathrm{~mol})$ and hydrazine hydrate $(0.5 \mathrm{~mL}, 0.01$ $\mathrm{mol}$ ) in $30 \mathrm{~mL}$ ethanol containing $0.1 \mathrm{~mL}$ piperidine was refluxed for $8 \mathrm{hr}$. The reaction mixture was concentrated under reduced pressure and the residue washed with acidified cold water and then triturated with methanol. The formed pale yellow product was filtered, washed well with methanol. Yield $66 \%$, m.p. $250^{\circ} \mathrm{C}-252^{\circ} \mathrm{C}$. IR: $1670(\mathrm{C}=\mathrm{O})$, $3282(\mathrm{NH})$ and $3432\left(\mathrm{NH}_{2}\right) .{ }^{1} \mathrm{H}$ NMR (DMSO): $4.82(s, \mathrm{br}$, $2 \mathrm{H}, \mathrm{NH}_{2}, \mathrm{D}_{2} \mathrm{O}$, exchangeable), $5.1(s, 1 \mathrm{H}, \mathrm{CH}-$ methine), 6.7 - $7.9(m, 11 \mathrm{H}, \mathrm{Ar}-\mathrm{H})$ and $8.85\left(s, \mathrm{br}, 1 \mathrm{H}, \mathrm{NH}, \mathrm{D}_{2} \mathrm{O}\right.$, exchangeable). MS: $m / z$ 309([M+3] $\left.]^{+}, 45 \%\right)$. Calcd for $\mathrm{C}_{17} \mathrm{H}_{14} \mathrm{~N}_{4} \mathrm{O}_{2}$ (306.32): C 66.66, H 4.61, N 18.29\%. Found: C 66.01, H 3.20, N $17.66 \%$.

Preparation of 3-amino-3-(3-amino-5-oxo-1-(4-oxo3-phenyl-3,4-dihydro-2H-chromeno[2,3- $d]$ pyrimidin-2yl)-1H-pyrazol-4(5H)-ylidene)propanenitrile (5), and its derivatives, $(7$ - 10, 12, 13a, b).

General procedure: To a solution of hydrazide 3 (3.06 g, $0.01 \mathrm{~mol})$ and ethyl 3-amino-2,4-dicyanobut-2-enoate (1.79 mL, $0.01 \mathrm{~mol})$ in $30 \mathrm{~mL}$ ethanol containing $0.1 \mathrm{~mL}$ piperidine was refluxed for $6 \mathrm{~h}$. then allowed to cool. The formed solid was filtered off, washed with methanol to afford the pyrazoile derivative 5. Analogously, diethyl malonate $(1.60 \mathrm{~mL}, 0.01 \mathrm{~mol})$, ethyl 2-cyanoacetate $(1.13 \mathrm{~mL})$, ethyl 3-oxobutanoate $(1.30 \mathrm{~mL})$, pentane-2,4-dione $(1.00$ $\mathrm{mL}), 2$-(ethoxymethylene) malononitrile (1.22 gm) and 2-cyano- $N$-phenylacetamide $(1.60 \mathrm{gm})$ were reacted with compound 3 to yield $(7-10,12,13 a, b)$, respectively.

3-Amino-3-(3-amino-5-oxo-1-(4-oxo-3-phenyl-3,4dihdro-2H-chromeno-[2,3- $d]$ pyrimidin-2-yl)-1H-pyrazol-4(5H)-ylidene)propanenitrile (5). Yellow crystals
$(\mathrm{MeOH})$, yield $70 \%$, m.p. $250^{\circ} \mathrm{C}-252^{\circ} \mathrm{C}$. IR: $1699(\mathrm{CO})$, $2219(\mathrm{CN}), 3422\left(\mathrm{NH}_{2}\right) .{ }^{1} \mathrm{H}$ NMR (DMSO): 2.9 (s, 2H, $\left.\mathrm{CH}_{2}\right), 3.5\left(s, 2 \mathrm{H}, \mathrm{NH}_{2}\right), 3.7\left(s, 2 \mathrm{H}, \mathrm{NH}_{2}\right), 6.7$ - $7.9(m, 10 \mathrm{H}$, Ar-H). MS: $m / z 439\left(\left[\mathrm{M}^{+}\right], 60 \%\right)$. Calcd for $\mathrm{C}_{23} \mathrm{H}_{17} \mathrm{~N}_{7} \mathrm{O}_{3}$ (439.43). C 62.87, H 3.90, N 22.31\%. Found: C 61.01, H 3.20 , N $21.89 \%$.

1-(3,4-dihydro-4-oxo-3-phenyl-2H-chromeno[2,3-d]pyrimidin-2-yl)pyrazo-lidine-3,5-dione, (7). Pale yellow crystals $(\mathrm{MeOH})$, yield $66 \%$. m.p. $280^{\circ} \mathrm{C}-282^{\circ} \mathrm{C}$. IR: $v\left(\mathrm{~cm}^{-1}\right) 1685-1705(\mathrm{C}=\mathrm{O}), 3212(\mathrm{NH}),{ }^{1} \mathrm{HNMR}\left(\mathrm{CDCl}_{3}\right)$ : $\delta 3.2\left(s, 2 \mathrm{H}, \mathrm{CH}_{2}\right), 5.8(s, 1 \mathrm{H}, \mathrm{CH}$-methine $), 8.1(\mathrm{~s}, 1 \mathrm{H}$, $\mathrm{NH}$ of pyrazole ring) and $6.1-7.8(m, 10 \mathrm{H}, \mathrm{Ar}-\mathrm{H})$. MS: $m / z 374$ ([M] $]^{+}, 55 \%$ ). Calcd for $\mathrm{C}_{20} \mathrm{H}_{14} \mathrm{~N}_{4} \mathrm{O}_{4}$ (374.35). Calcd: C 64.17, H 3.77, N 14.97\%. Found: C 63.33, H 2.91, N 14.15\%.

Compound 5. Yield $70 \%$, m.p. $257^{\circ} \mathrm{C}-259^{\circ} \mathrm{C}$. IR: 1699 (CO), 2219 (CN), $3422\left(\mathrm{NH}_{2}\right) .{ }^{1} \mathrm{H}$ NMR (DMSO): $2.9\left(s, 2 \mathrm{H}, \mathrm{CH}_{2}\right), 3.5\left(s, 2 \mathrm{H}, \mathrm{NH}_{2}\right), 3.7\left(s, 2 \mathrm{H}, \mathrm{NH}_{2}\right), 6.7$ $7.9(m, 10 \mathrm{H}, \mathrm{Ar}-\mathrm{H})$. MS: $m / z 439\left(\left[\mathrm{M}^{+}\right], 60 \%\right)$. Calcd for $\mathrm{C}_{23} \mathrm{H}_{17} \mathrm{~N}_{7} \mathrm{O}_{3}$ (439.43). C 62.87, H 3.90, N $22.31 \%$. Found: C 61.01, H 3.20, N 21.89\%.

Compound 8. Pale green crystals (Ethanol), yield 50\%, m.p. $250^{\circ} \mathrm{C}-252^{\circ} \mathrm{C}$, IR: $v\left(\mathrm{~cm}^{-1}\right) 1685-1705(\mathrm{C}=\mathrm{O}), 3432$ $\left(\mathrm{NH}_{2}\right)$, MS: $m / z 373\left([\mathrm{M}]^{+}, 65 \%\right)$. Calcd for $\mathrm{C}_{20} \mathrm{H}_{15} \mathrm{~N}_{5} \mathrm{O}_{3}$ (373.36). Calcd: C 64.34, H 4.05, N 18.76\%. Found: C 63.33, H 3.91, N 17.15\%.

Compound 9. Brown crystals (Ethanol), yield 55\%, m.p. $190^{\circ} \mathrm{C}-192^{\circ} \mathrm{C}$. IR: $v\left(\mathrm{~cm}^{-1}\right) 1685-1705(\mathrm{C}=\mathrm{O})$, ${ }^{1} \mathrm{HNMR}\left(\mathrm{CDCl}_{3}\right): \delta 1.1\left(s, 3 \mathrm{H}, \mathrm{CH}_{3}\right), 2.5\left(s, 2 \mathrm{H}, \mathrm{CH}_{2}\right), 5.8$ ( $s, 1 \mathrm{H}, \mathrm{CH}-m e t h i n e), 6.7-7.9(m, 10 \mathrm{H}, \mathrm{Ar}-\mathrm{H})$. MS: $m / z$ 370 ([M-2] $\left.]^{+}, 60 \%\right)$. Calcd for $\mathrm{C}_{23} \mathrm{H}_{16} \mathrm{~N}_{4} \mathrm{O}_{3}$ (372.38). Calcd: C 67.73, H 4.33, N 15.05\%. Found: C 67.17, H 3.31, N $14.15 \%$.

Compound 10. Pale green crystals (Ethanol), yield 59 \%, m.p. $270^{\circ} \mathrm{C}-2^{\circ} \mathrm{C}$. IR: v $\left(\mathrm{cm}^{-1}\right) 1685-1705(\mathrm{C}=\mathrm{O})$, MS: $m / z 369$ ([M-1] $]^{+}, 66 \%$ ). Calcd for $\mathrm{C}_{22} \mathrm{H}_{18} \mathrm{~N}_{4} \mathrm{O}_{2}$ (370.4). Calcd: C 71.34, H 4.90, N 15.13\%. Found: C 70.17, H 3.31 , N 14.15\%.

Preparation of 1-phenyl-tetrazolo[4',5':2,3] pyimido[4,5-b]chromen-12-one, (11).

To a stirred cold solution of $\mathbf{3}(0.306 \mathrm{~g}, 0.001 \mathrm{~mol})$ in 30 $\mathrm{mL}$ of glacial acetic acid, a cold solution of sodium nitrite $(0.7 \mathrm{~g}, 0.01 \mathrm{~mol})$ in $10 \mathrm{~mL}$ of $\mathrm{H}_{2} \mathrm{O}$ was added drop wise stirring at $5^{\circ} \mathrm{C}$. The mixture was stirred for further four hours at room temperature. The solid that precipitated was collected by filtration, washed with water and air dried to afford 55\% yield of the tetrazolo derivative 11. Yield 55\%, m.p. $250^{\circ} \mathrm{C}-252^{\circ} \mathrm{C}$. IR: 1699 (CO). MS: $m / z 317\left(\left[\mathrm{M}^{+}\right]\right.$, $60 \%$ ). Calcd for $\mathrm{C}_{17} \mathrm{H}_{11} \mathrm{~N}_{5} \mathrm{O}_{2}$ (317.3). C 64.35, H 3.49, N 22.07\%. Found: C 63.01, H 2.20, N 21.89\%.

Compound 12. Yellow crystals (Ethanol), yield 55\%, m.p. $259^{\circ} \mathrm{C}-261^{\circ} \mathrm{C}$. IR: $v\left(\mathrm{~cm}^{-1}\right) 1685-1705(\mathrm{C}=\mathrm{O}), 2219$ (CN), 3212 - $3423\left(\mathrm{NH}_{2}\right), \mathrm{MS}: \mathrm{m} / z 382\left([\mathrm{M}+1]^{+}, 50 \%\right)$. Calcd for $\mathrm{C}_{21} \mathrm{H}_{14} \mathrm{~N}_{6} \mathrm{O}_{2}$ (382.37). Calcd: C 65.96, H 3.69, N 
21.98\%. Found: C 64.17, H 2.31, N 20.15\%.

Compound 13a. Yellow crystals (Ethanol), yield 62\%, m.p. $150^{\circ} \mathrm{C}-152^{\circ} \mathrm{C}$. IR: $v\left(\mathrm{~cm}^{-1}\right) 1685-1705(\mathrm{C}=\mathrm{O}), 3212-$ $3432\left(\mathrm{NH}_{2}\right),{ }^{1} \mathrm{HNMR}\left(\mathrm{CDCl}_{3}\right): \delta 4.1\left(s, 2 \mathrm{H}, \mathrm{NH}_{2}\right), 4.3(s$, 1H, NHPh), 6.2 (s, 1H, = CH-), 6.7 - 7.9 (m, 10H, Ar-H). MS: $m / z$ 448([M] $\left.]^{+}, 40 \%\right)$. Calcd for $\mathrm{C}_{26} \mathrm{H}_{20} \mathrm{~N}_{6} \mathrm{O}_{2}$ (448.48). Calcd: C 69.63, H 4.49, N 18.74\%. Found: C 68.17, H 3.31 , N $17.15 \%$.

Compound 13b. Pale yellow crystals $(\mathrm{MeOH})$, yield $50 \%$, m.p. $180^{\circ} \mathrm{C}-182^{\circ} \mathrm{C}$. IR: $v\left(\mathrm{~cm}^{-1}\right) 1685-1705(\mathrm{C}=\mathrm{O})$, 3212 - $3432\left(\mathrm{NH}_{2}\right), \mathrm{MS}: \mathrm{m} / z 456\left([\mathrm{M}+1]^{+}, 35 \%\right)$. Calcd for $\mathrm{C}_{23} \mathrm{H}_{17} \mathrm{~N}_{7} \mathrm{O}_{2} \mathrm{~S}$ (455.49). Calcd: $\mathrm{C} 60.65, \mathrm{H} \mathrm{3.76,} \mathrm{N}$ 71.53, S 7.04\%. Found: C 59.17, H 2.66, N 70.26, S 6.54\%.

Preparation of 2-(2-benzylidenehydrazinyl)-3-phenyl3H-chromeno[2,3- $d$ ]pyri-midin-4(10H)-one, (14). A mixture of the 2-hydrazinyl-3-phenyl-2H-chromeno [2,3- $d]$ pyrimidin-4(3H)-one 3 (3.06 g, $0.01 \mathrm{~mol})$ and benzaldehyde $(1.06,0.01 \mathrm{~mol})$ in $20 \mathrm{~mL}$ ethanol containing $0.1 \mathrm{~mL}$ of piperidine was refluxed for $8 \mathrm{hr}$. The reaction mixture was concentrated, cooled then poured into ice $/ \mathrm{H}_{2} \mathrm{O}$ mixture the solid product thus so formed was filtered, washed for several times with water to afford $14(1.66 \mathrm{~g}, 61 \%)$ as a brown solid; m.p. $225^{\circ} \mathrm{C}-227^{\circ} \mathrm{C}$. IR: $v\left(\mathrm{~cm}^{-1}\right) 1705(\mathrm{C}=\mathrm{O})$, $3322(\mathrm{NH}) .{ }^{1} \mathrm{HNMR}\left(\mathrm{CDCl}_{3}\right): \delta 6.7-7.9(\mathrm{~m}, 14 \mathrm{H}, \mathrm{Ar}-\mathrm{H})$, $8.11(s, 1 \mathrm{H}, \mathrm{CH}=)$ and $10.11(s, 1 \mathrm{H}, \mathrm{NH}) . \mathrm{MS}: m / z 397$ $\left([\mathrm{M}]^{+}, 65 \%\right)$. Calcd for $\mathrm{C}_{24} \mathrm{H}_{18} \mathrm{~N}_{4} \mathrm{O}_{2}$ (394.43). Calcd: $\mathrm{C}$ 73.08, H 4.60, N $14.20 \%$. Found: C 72.14, H 4.01, N $13.88 \%$.

Preparation of 5-oxo-1-(4-oxo-3-phenyl-4, 10-dihydro-3H-chromeno[2,3- $d$ ]pyrimidin-2-yl)-3-phenylpyra zolidine-4-carbonitrile, (16). A solution of benzylidenehydrazinyl 14 (3.94 g, $0.01 \mathrm{~mol})$ and ethyl 2-cyanoacetate $(1.13 \mathrm{~mL})$ in $30 \mathrm{~mL}$ ethanol containing $0.1 \mathrm{~mL}$ piperidine was refluxed for $6 \mathrm{~h}$. then allowed to cool. The formed solid was filtered off, washed with methanol to afford the pyrazole derivative $16(2.45 \mathrm{~g}, 61 \%)$ as a brown solid; m.p. $295^{\circ} \mathrm{C}-297^{\circ} \mathrm{C}$. IR: $v\left(\mathrm{~cm}^{-1}\right) 1670(\mathrm{C}=\mathrm{O}), 2217(\mathrm{CN}), 3432$ (NH). ${ }^{1} \mathrm{HNMR}\left(\mathrm{CDCl}_{3}\right): \delta 3.98(s, 1 \mathrm{H}, \mathrm{NH}), 4.12(s, 1 \mathrm{H}$, C-3 pyrazole), $4.21(\mathrm{~s}, 1 \mathrm{H}, \mathrm{C}-4$ pyrazole $)$ and $6.7-7.9(\mathrm{~m}$, 14H, Ar-H). MS: $m / z 462\left([\mathrm{M}+1]^{+}, 55 \%\right)$. Calcd for $\mathrm{C}_{27} \mathrm{H}_{19} \mathrm{~N}_{5} \mathrm{O}_{3}$ (461.47). Calcd: $\mathrm{C} 70.27$, H 4.15, N $15.18 \%$. Found: C 69.12, H 4.01, N 14.11\%.

Preparation of 5-chloro-1-(4-oxo-3-phenyl-4,10-dihydro-3H-chromeno[2,3-d]pyrimidin-2-yl)-3-phenyl-2, 3dihydro-1H-pyrazole-4-carbonitrile, (17). A suspension of $1.0 \mathrm{~g}$ of $\mathbf{1 6}$ in $10 \mathrm{ml}$ of phosphoryl chloride was heated at $90^{\circ} \mathrm{C}$ for $5 \mathrm{hr}$ and, after cooling, crushed ice was added. The solution was then made basic with concentrated ammonium hydroxide $(\mathrm{pH}=8)$ and the solid that precipitated was collected by filtration, washed with water and purified by crystallization to give $\mathbf{1 7}$ as a Yellow solid; m.p. $189^{\circ} \mathrm{C}-191^{\circ} \mathrm{C}$. IR: $v\left(\mathrm{~cm}^{-1}\right) 1700(\mathrm{C}=\mathrm{O}), 2217(\mathrm{CN})$, $3425(\mathrm{NH}) .{ }^{1} \mathrm{HNMR}\left(\mathrm{CDCl}_{3}\right): \delta 3.98(s, 1 \mathrm{H}, \mathrm{NH}), 4.61(s$, $1 \mathrm{H}, \mathrm{C}-3$ pyrazole) and $6.7-7.9(m, 14 \mathrm{H}, \mathrm{Ar}-\mathrm{H})$. MS: $m / z$
$478\left([\mathrm{M}-1]^{+}, 16 \%\right)$. Calcd for $\mathrm{C}_{27} \mathrm{H}_{18} \mathrm{~N}_{5} \mathrm{O}_{2} \mathrm{Cl}$ (479.92). Calcd: C 67.57, H 3.78, N 14.59\%. Found: C 66.12, H 3.21, N 14.51\%.

Preparation of 2-(4-amino-3-phenyl-2, 3-dihydro$1 H$-pyrazolo[4,3-e][1, 2, 4]-triazolo[1,5-a] pyrimidin1-yl)-3-phenyl-3H-chromeno[2,3- $d]$ pyrimidin-4(10H)one, (18). Amixtuer of 17 (4.79 g, $0.01 \mathrm{~mol})$ and 5amino-1,2,4-1H-triazole $(0.84 \mathrm{~g})$ in $30 \mathrm{~mL}$ ethanol containing $0.1 \mathrm{~mL}$ piperidine was refluxed for $3 \mathrm{hr}$. then allowed to cool. The formed brownish solid was filtered off, washed with methanol to afford the pyrazoile derivative $18(2.4 \mathrm{~g}, 50 \%)$ as a brownish solid; m.p. $156^{\circ} \mathrm{C}$ $158^{\circ} \mathrm{C}$. IR: v $\left(\mathrm{cm}^{-1}\right) 1670(\mathrm{C}=\mathrm{O}), 3432(\mathrm{NH}) .{ }^{1} \mathrm{HNMR}$ (DMSO): $\delta 3.98(s$, br, 1H, NH), $5.21(s, 1 \mathrm{H}, \mathrm{C}-3$ pyrazole), $6.13\left(s, \mathrm{br}, 2 \mathrm{H}, \mathrm{NH}_{2}\right)$ and $6.7-7.9(m, 14 \mathrm{H}, \mathrm{Ar}-\mathrm{H})$ and $8.71(\mathrm{~s}, 1 \mathrm{H}$, methine proton of triazole ring). MS: $\mathrm{m} / \mathrm{z}$ $527\left([\mathrm{M}]^{+}, 25 \%\right)$. Calcd for $\mathrm{C}_{29} \mathrm{H}_{21} \mathrm{~N}_{9} \mathrm{O}_{2}$ (527.54). Calcd: C 66.03, H 4.01, N 23.90 \%. Found: C 65.31, H 3.22, N $22.84 \%$.

Preparation of 4-amino-7-(methylthio)-1-(4-oxo-3phenyl-4,10-dihydro-3H-chromeno[2,3- $d]$ pyrimidin-2yl)-3-phenyl-2,3-dihydro-1H-dipyrazolo [1,5-a:4',3'-e] pyrimidine-6-carbonitrile, (19). Amixtuer of 17 (4.79 g, $0.01 \mathrm{~mol}$ ) and 5-amino-3-(methylthio)- $1 H$-pyrazole-4- carbonitrile $(1.54 \mathrm{~g}, 0.01 \mathrm{~mol})$ in $30 \mathrm{~mL}$ ethanol containing $0.1 \mathrm{~mL}$ piperidine was refluxed for $5 \mathrm{~h}$. then allowed to cool. The formed pale yellow solid was filtered off, washed with methanol to afford the pyrazoile derivative $19(2.4 \mathrm{~g}, 50 \%)$ as a pale yellow solid $(\mathrm{MeOH})$; m.p. $240^{\circ} \mathrm{C}-$ $242^{\circ} \mathrm{C}$. IR: $v\left(\mathrm{~cm}^{-1}\right) 1670(\mathrm{C}=\mathrm{O}), 2221(\mathrm{CN}), 3432(\mathrm{NH})$. ${ }^{1} \mathrm{HNMR}$ (DMSO): $\delta 3.54\left(s, 3 \mathrm{H}, \mathrm{SCH}_{3}\right), 3.98(s, \mathrm{br}, 1 \mathrm{H}$, $\mathrm{NH}), 5.21$ ( $s, 1 \mathrm{H}, \mathrm{C}-3$ pyrazole), $6.13\left(s, \mathrm{br}, 2 \mathrm{H}, \mathrm{NH}_{2}\right)$ and $6.7-7.9(\mathrm{~m}, 14 \mathrm{H}, \mathrm{Ar}-\mathrm{H})$ and $8.71(\mathrm{~s}, 1 \mathrm{H}$, methine proton of triazole ring). MS: $m / z 597$ ([M] $\left.]^{+}, 35 \%\right)$. Calcd for $\mathrm{C}_{32} \mathrm{H}_{23} \mathrm{~N}_{9} \mathrm{O}_{2} \mathrm{~S}$ (597.65). Calcd: C 64.31, H 3.88, N 21.09 \%. Found: C 63.56, H 2.52, N 20.78\%.

Preparation 5-methoxy-1-(4-oxo-3-phenyl-4, 10-dihydro-3H-chromeno[2,3-d]py-rimidin-2-yl)-3-phenyl2,3-dihydro-1H-pyrazole-4-carbonitrile, (20). A solution of $10 \mathrm{mmol}$ of freshly prepared sodium methoxide and $1.0 \mathrm{mmol}$ of the chloro derivatives $17 \mathrm{in} 10 \mathrm{ml}$ of anhydrous methanol was refluxed for $4 \mathrm{hr}$ then the reaction mixture was evaporated to dryness in vacuo. The crude residue was treated with water and neutralized with $10 \%$ hydrochloric acid, and the solid precipitate, collected by filtration, was purified by crystallization to obtain $\mathbf{2 0}$ as yellow crystals (DMSO-HCl); yield $30 \%$. m.p. $288^{\circ} \mathrm{C}$ 290 C. IR: $v\left(\mathrm{~cm}^{-1}\right) 1699(\mathrm{C}=\mathrm{O}), 2219(\mathrm{CN}), 3422(\mathrm{NH})$. ${ }^{1} \mathrm{HNMR}$ (DMSO): $\delta 3.82\left(s, 3 \mathrm{H}, \mathrm{OCH}_{3}\right), 3.98(s, \mathrm{br}, 1 \mathrm{H}$, $\mathrm{NH}), 4.61(s, 1 \mathrm{H}, \mathrm{C}-3$ pyrazole $)$ and $6.7-7.9(m, 14 \mathrm{H}$, Ar-H). MS: $m / z 475$ ([M] $\left.]^{+}, 62 \%\right)$. Calcd for $\mathrm{C}_{28} \mathrm{H}_{23} \mathrm{~N}_{9} \mathrm{O}_{2} \mathrm{~S}$ (597.65). Calcd: C 64.31, H 3.88, N 21.09 \%. Found: C 63.56, H 2.52, N 20.78\%.

Preparation 5-azido-1-(4-oxo-3-phenyl-4, 10-dihy- 
dro-3H-chromeno[2,3- $d$ ]pyri-midin-2-yl)-3-phenyl-2,3 -dihydro-1H-pyrazole-4-carbonitrile, (21). A solution of the chloro compound $\mathbf{1 7}(1.437 \mathrm{~g}, 3 \mathrm{mmol})$ in acetone $(5 \mathrm{~mL})$ was stirred and ice-cooled. The solution of $\mathrm{NaN}_{3}$ $(0.13 \mathrm{~g}, 2 \mathrm{mmol})$ in water $(1 \mathrm{~mL})$ was added drop wise in the solution and this mixture was stirred for $1 \mathrm{hr}$ at room temperature. After evaporation of acetone, the crude product was separated by filtration and recrystallized from dichlormethane. We have obtained $(0.9 \mathrm{~g}, 62 \%)$ of 21 as Yellow solid with $\mathrm{mp} 150^{\circ} \mathrm{C}-152^{\circ} \mathrm{C}[21,22]$. IR: $v\left(\mathrm{~cm}^{-1}\right)$ $1699(\mathrm{C}=\mathrm{O}), 2217(\mathrm{CN}), 3422(\mathrm{NH}) .{ }^{1} \mathrm{HNMR}$ (DMSO): $\delta$ $3.98(s, \mathrm{br}, 1 \mathrm{H}, \mathrm{NH}), 4.61(s, 1 \mathrm{H}, \mathrm{C}-3$ pyrazole $)$ and $6.7-$ 7.9 ( $m, 14 \mathrm{H}, \mathrm{Ar}-\mathrm{H})$. MS: $m / z 486\left([\mathrm{M}]^{+}, 32 \%\right)$. Calcd for $\mathrm{C}_{27} \mathrm{H}_{18} \mathrm{~N}_{8} \mathrm{O}_{2}$ (486.48). Calcd: C 66.66, H 3.73, N 23.03\%. Found: C 65.76, H 2.56, N 22.73\%.

Preparation of 2-(4-amino- 3-phenyl-2,3-dihydropyrazolo[3,4-c]pyrazol-1(6H)-yl)-3-phenyl-3H-chromeno[2,3- $d$ ]pyrimidin-4(10H)-one, (23). A mixture of $17(3.20 \mathrm{~g}, 0.01 \mathrm{~mol})$ and hydrazine hydrate $(0.5 \mathrm{~mL}, 0.01$ mol) in $20 \mathrm{~mL}$ DMF containing $0.1 \mathrm{~mL}$ piperidine was refluxed for $8 \mathrm{hr}$. The reaction mixture was concentrated under reduced pressure and the residue washed with acidified cold water and then triturated with methanol. The formed yellow product was filtered, washed well with methanol. Yield $58 \%$, m.p. $150^{\circ} \mathrm{C}-152^{\circ} \mathrm{C}$. IR: 1670 $(\mathrm{C}=\mathrm{O}), 3432(\mathrm{NH})$ and $3455\left(\mathrm{NH}_{2}\right) .{ }^{1} \mathrm{H}$ NMR (DMSO): $3.98(s, \mathrm{br}, 1 \mathrm{H}, \mathrm{NH}), 5.21$ ( $s, 1 \mathrm{H}, \mathrm{CH}-$ methine), 6.7 - 7.9 $(m, 11 \mathrm{H}, \mathrm{Ar}-\mathrm{H})$ and $9.21(s, 1 \mathrm{H}, \mathrm{NH})$. MS: $m / z$ 475([M] ${ }^{+}$, 64\%). Calcd for $\mathrm{C}_{27} \mathrm{H}_{21} \mathrm{~N}_{7} \mathrm{O}_{2}$ (475.50): C 68.20, H 4.45, N 20.62\%. Found: C 67.03, H 3.32, N 19.23\%.

Preparation of 3-(2-imino-2 $H$ - chromen-3-yl)-1-phenyl-3-(phenylamino) prop-2-en-1-one, (24a) and its derivatives (24b-e).

General procedure: A mixture of the 2-imino- $N$ phenyl-2H-chromene-3-carboxamide 1 (2.64 g, $0.01 \mathrm{~mol})$ and acetophenone $(1.2 \mathrm{ml}, 0.01 \mathrm{~mol}), p$-hydroxyacetophenone (1.36 g, $0.01 \mathrm{~mol}), p$-nitroacetophenone (1.65 g, 0.01 $\mathrm{mol}), o$-nitroacetophenone $(1.65 \mathrm{~g}, 0.01 \mathrm{~mol})$ and $p$-chloroacetophenone $(1.54 \mathrm{~g}, 0.01 \mathrm{~mol})$ respectively, in $20 \mathrm{~mL}$ DMF containing $0.1 \mathrm{ml}$ of piperidine was refluxed for $8 \mathrm{hr}$. The reaction mixture was concentrated, cooled then poured into ice $/ \mathrm{H}_{2} \mathrm{O}$ mixture the solid product thus so formed was filtered, washed for several times with water to afford 24a 24e derivatives.

Compound 24a. Yellow crystals $(\mathrm{MeOH})$, yield $60 \%$. m.p. $182^{\circ} \mathrm{C}-184^{\circ} \mathrm{C}$. IR: $v\left(\mathrm{~cm}^{-1}\right) 1665-1705(\mathrm{C}=\mathrm{O}), 3322$ (NH), ${ }^{1} \mathrm{HNMR}$ (DMSO): $\delta 4.1$ ( $s, 1 \mathrm{H}, \mathrm{NH}-\mathrm{m}$-ane), $6.5(s$, $1 \mathrm{H}$, CH-ethylene), 7.1-7.6 (m, 15H, Ar-H), $11.5(s, 1 \mathrm{H}$, $=\mathrm{NH})$. MS: $m / z 366\left([\mathrm{M}]^{+}, 40 \%\right)$. Calcd for $\mathrm{C}_{24} \mathrm{H}_{18} \mathrm{~N}_{2} \mathrm{O}_{2}$ (366.41). Calcd: C 78.67, H 4.95, N 7.65\%. Found: C $77.17, \mathrm{H} 4.01$, N 8.15\%.

Compound 24b. Pale green crystals $(\mathrm{MeOH})$, yield $55 \%$, m.p. $220^{\circ} \mathrm{C}-222^{\circ} \mathrm{C}$. IR: $v\left(\mathrm{~cm}^{-1}\right) 1665-1705(\mathrm{C}=\mathrm{O})$, $3445(\mathrm{OH}),{ }^{1} \mathrm{HNMR}\left(\mathrm{CDCl}_{3}\right): \delta 4.1(s, 1 \mathrm{H}, \mathrm{NH}-\mathrm{amine})$,
6.5 ( $s, 1 \mathrm{H}, \mathrm{CH}$-ethylene), 7.1 - 7.6 ( $m, 14 \mathrm{H}, \mathrm{Ar}-\mathrm{H}), 11.5(s$, $1 \mathrm{H},=\mathrm{NH})$ and $9.9(\mathrm{~s}, 1 \mathrm{H}$, phenolic $\mathrm{OH})$. MS: $m / z 383([\mathrm{M}+$ $1]^{+}, 60 \%$ ). Calcd for $\mathrm{C}_{24} \mathrm{H}_{18} \mathrm{~N}_{2} \mathrm{O}_{3}$ (382.41). Calcd: $\mathrm{C} 75.38$, H 4.74, N 7.33\%. Found: C 74.17, H 3.91, N 6.15\%.

Compound 24c. Brown crystals (Ethanol), yield 65\%, m.p. $190^{\circ} \mathrm{C}-192^{\circ} \mathrm{C}$. IR: $v\left(\mathrm{~cm}^{-1}\right) 1665-1705(\mathrm{C}=\mathrm{O})$, ${ }^{1} \mathrm{HNMR}$ (DMSO): $\delta 4.1(s, 1 \mathrm{H}, \mathrm{NH}-a m i n e), 6.5(s, 1 \mathrm{H}$, CH-ethylene), $7.1-7.6(m, 14 \mathrm{H}, \mathrm{Ar}-\mathrm{H}), 11.5(s, 1 \mathrm{H},=\mathrm{NH})$. MS: $m / z 411\left([\mathrm{M}]^{+}, 62 \%\right)$. Calcd for $\mathrm{C}_{24} \mathrm{H}_{17} \mathrm{~N}_{3} \mathrm{O}_{4}$ (411.41). Calcd: C 70.07, H 4.16, N 10.21\%. Found: C 69.17, H $3.31, \mathrm{~N} 9.15 \%$.

Compound 24d. Brown crystals (Ethanol), yield $59 \%$, m.p. $170^{\circ} \mathrm{C}-172^{\circ} \mathrm{C}$. IR: $v\left(\mathrm{~cm}^{-1}\right) 1665-1705(\mathrm{C}=\mathrm{O})$. MS: $m / z 410$ ([M-1] $\left.]^{+}, 52 \%\right)$. Calcd for $\mathrm{C}_{24} \mathrm{H}_{17} \mathrm{~N}_{3} \mathrm{O}_{4}$ (411.41). Calcd: C70.07, H 4.16, N 10.21\%. Found: C 69.17, H 3.31 , N 9.15\%.

Compound 24e. Yellow crystals (Ethanol), yield 55\%, m.p. $255^{\circ} \mathrm{C}-257^{\circ} \mathrm{C}$. IR: v $\left(\mathrm{cm}^{-1}\right) 1665-1705(\mathrm{C}=\mathrm{O})$, ${ }^{1} \mathrm{HNMR}$ (DMSO): $\delta 4.1(s, 1 \mathrm{H}, \mathrm{NH}-\mathrm{amine}), 6.5(s, 1 \mathrm{H}$, CH-ethylene), 7.1 - $7.6(m, 14 \mathrm{H}, \mathrm{Ar}-\mathrm{H}), 11.5(s, 1 \mathrm{H},=\mathrm{NH})$. MS: $m / z 400\left([\mathrm{M}]^{+}, 60 \%\right)$. Calcd for $\mathrm{C}_{24} \mathrm{H}_{17} \mathrm{~N}_{2} \mathrm{O}_{2} \mathrm{Cl}(400.86)$. Calcd: C 71.91, H 4.27, N 6.99, C18.84\%. Found: C 70.17, H 3.31, N 5.15, Cl7.18\%.

Preparation of 2-imino- $N, N^{\prime}$-diphenyl-2 $H$-chromene-3-carboxamidines, (25a) and its derivatives (25b25e).

General procedure: A mixture of equimolar amount of compound $\mathbf{1}(2.64 \mathrm{~g}, 0.01 \mathrm{~mol})$ and aniline $(0.93 \mathrm{ml}, 0.01$ mol), $p$-hydroxyaniline $(1.09 \mathrm{~g}, 0.01 \mathrm{~mol}), p$-nitroaniline $(1.38 \mathrm{~g}, 0.01 \mathrm{~mol}), o$-nitroaniline $(1.38 \mathrm{~g}, 0.01 \mathrm{~mol}$ and p-chloroaniline $(1.27 \mathrm{~g}, 0.01 \mathrm{~mol})$, respectively in $30 \mathrm{~mL}$ ethanol containing $0.1 \mathrm{~mL}$ of piperidine was refluxed for 5 $\mathrm{hr}$. The reaction mixture was concentrated, poured into ice $/ \mathrm{H}_{2} \mathrm{O}$ mixture, and the solid product thus formed, filtered, washed for several times with water and crystallized from methanol.

Compound 25a. Pale brown crystals (Methanol), yield $58 \%$, m.p. $205^{\circ} \mathrm{C}-207^{\circ} \mathrm{C}$. IR: $v\left(\mathrm{~cm}^{-1}\right) 3380(\mathrm{NH}),{ }^{1} \mathrm{HNMR}$ $\left(\mathrm{CDCl}_{3}\right): \delta 4.1(s, 1 \mathrm{H}, \mathrm{NH}-$ amine $), 7.1-7.6(\mathrm{~m}, 15 \mathrm{H}, \mathrm{Ar}-$ $\mathrm{H}), 11.5(\mathrm{~s}, 1 \mathrm{H},=\mathrm{NH})$. MS: $\mathrm{m} / z 339\left([\mathrm{M}]^{+}, 65 \%\right)$. Calcd for $\mathrm{C}_{22} \mathrm{H}_{17} \mathrm{~N}_{3} \mathrm{O}(339.39)$. C 77.86, H 5.05, N $12.38 \%$. Found: C 76.66, H 4.52, N 11.12\%.

Compound 25b. Yellow crystals (Methanol), yield 60\%, m.p. $210^{\circ} \mathrm{C}-212^{\circ} \mathrm{C}$. IR: v $\left(\mathrm{cm}^{-1}\right) 3380(\mathrm{NH}), 3445(\mathrm{OH})$, ${ }^{1} \mathrm{HNMR}$ (DMSO): $\delta 4.1(s, 1 \mathrm{H}, \mathrm{NH}$-amine), 7.1- 7.6 (m, $14 \mathrm{H}, \mathrm{Ar}-\mathrm{H}), 11.5(s, 1 \mathrm{H},=\mathrm{NH})$ and $9.9(\mathrm{~s}, 1 \mathrm{H}$, phenolic OH). MS: $m / z 357\left([\mathrm{M}+2]^{+}, 50 \%\right)$. Calcd for $\mathrm{C}_{22} \mathrm{H}_{17} \mathrm{~N}_{3} \mathrm{O}_{2}$ (355.39). C 74.35, H 4.82, N 11.82\%. Found: C 73.45, H $3.52, \mathrm{~N} 10.12 \%$.

Compound 25c. Pale yellow crystals (Methanol), yield $64 \%$, m.p. $285^{\circ} \mathrm{C}-287^{\circ} \mathrm{C} .{ }^{1} \mathrm{HNMR}$ (DMSO): $\delta 4.1(s, 1 \mathrm{H}$, NH-amine), $7.1-7.6(m, 14 \mathrm{H}, \mathrm{Ar}-\mathrm{H}), 11.5(s, 1 \mathrm{H},=\mathrm{NH})$, MS: $m / z 385\left([\mathrm{M}+1]^{+}, 55 \%\right)$. Calcd for $\mathrm{C}_{22} \mathrm{H}_{16} \mathrm{~N}_{4} \mathrm{O}_{3} \mathrm{Cl}$ (384.39). C 68.74, H 4.20, N 14.58\%. Found: C 67.72, H 


\subsection{2, N 13.12\%.}

Compound 25d. Yellow crystals (Methanol), yield 60\%, m.p. $215^{\circ} \mathrm{C}-117^{\circ} \mathrm{C}$. MS: $m / z$ 384([M] $\left.]^{+}, 55 \%\right)$. Calcd for $\mathrm{C}_{22} \mathrm{H}_{16} \mathrm{~N}_{4} \mathrm{O}_{3} \mathrm{Cl}$ (384.39). C 68.74, H 4.20, N 14.58\%. Found: C 67.72, H 3.52, N 13.12\%.

Compound 25e. Pale brown crystals (methanol), yield $55 \%$, m.p. $230^{\circ} \mathrm{C}-232^{\circ} \mathrm{C}$. ${ }^{1} \mathrm{HNMR}$ (DMSO): $\delta 4.1(s, 1 \mathrm{H}$, NH-amine), 7.1 - $7.6(m, 14 \mathrm{H}, \mathrm{Ar}-\mathrm{H}), 11.5(\mathrm{~s}, 1 \mathrm{H},=\mathrm{NH})$, MS: $m / z 372\left([\mathrm{M}-1]^{+}, 70 \%\right)$. Calcd for $\mathrm{C}_{22} \mathrm{H}_{16} \mathrm{~N}_{3} \mathrm{OCl}$ (373.83). C 70.68, H 4.31, N 11.24, Cl 9.48\%. Found: C 69.45, H 3.52, N 11.12, Cl 8.48\%.

Preparation of 4,5-dihydro-5-(2-imino-2H-chromen3-yl)- $N$,3-diphenyl-1 $H$-pyrazol-5-amine, (26a) a- nd its derivatives (26b and 27).

General procedure: A mixture of 24a (3.66 g, 0.01 mol) and hydrazine hydrate $(0.05 \mathrm{ml}, 0.01 \mathrm{~mol})$, phenyl hydrazine $(1.08 \mathrm{ml}, 0.01 \mathrm{~mol})$ and hydroxylamine hydrochloride $(0.69 \mathrm{gm}, 0.01 \mathrm{~mol})$ containing $0.1 \mathrm{ml}$ piperidine was refluxed for $8 \mathrm{hr}$. The reaction mixture was concentrated under reduced pressure and the residue washed with acidified cold water and then triturated with methanol, and the solid product thus formed, filtered, washed for several times with water and crystallized from methanol.

Compound 26a. Pale brown crystals (methanol), yield $66 \%$, m.p. $155^{\circ} \mathrm{C}-157^{\circ} \mathrm{C}$. IR: $3117-3293(\mathrm{NH}) \cdot 1 \mathrm{H}$ NMR (DMSO): 2.9 (s, 2H, $\left.\mathrm{CH}_{2}\right), 4.1$ (s, 1H, NH-amine), 7.1 (s, H, NH hydrazide), 7.2 - 7.8 (m, 15H, Ar-H), 11.5 (s, $1 \mathrm{H},=\mathrm{NH})$. MS: $\mathrm{m} / \mathrm{z} 383\left([\mathrm{M}+3]^{+}, 55 \%\right)$. Calcd for $\mathrm{C}_{24} \mathrm{H}_{20} \mathrm{~N}_{4} \mathrm{O}$ (380.44): C 75.77, H 5.30, N 14.73\%. Found: $\mathrm{C}$ 74.01, H 4.20, N 13.66\%.

Compound 26b. Brown crystals (Methanol), yield 50\%, m.p. $295^{\circ} \mathrm{C}-297^{\circ} \mathrm{C}$. IR: 3117 - $3293(\mathrm{NH})$. $1 \mathrm{H}$ NMR (DMSO): 1.9 (s, 2H, CH2), 4.1 (s, 1H, NH-amine), 7.1 (s, $\mathrm{H}, \mathrm{NH}$ hydrazide), $7.2-7.8(\mathrm{~m}, 20 \mathrm{H}, \mathrm{Ar}-\mathrm{H})$ and 11.5 (s, $1 \mathrm{H},=\mathrm{NH})$. MS: m/z $456([\mathrm{M}+], 75 \%)$. Calcd for $\mathrm{C}_{30} \mathrm{H}_{24} \mathrm{~N}_{4} \mathrm{O}$ (456.54): C 78.92, H 5.30, N 12.27\%. Found: C 77.01, H 4.20, N $11.66 \%$.

Compound 27. Pale brown crystals (Methanol), yield $55 \%$, m.p. $230^{\circ} \mathrm{C}-232^{\circ} \mathrm{C}$. IR: 3117 - 3293 (NH). ${ }^{1} \mathrm{H}$ NMR (DMSO): $1.9\left(s, 2 \mathrm{H}, \mathrm{CH}_{2}\right), 4.1(s, 1 \mathrm{H}, \mathrm{NH}$-amine $), 7.2$ $7.8(m, 15 \mathrm{H}, \mathrm{Ar}-\mathrm{H})$ and $11.5(s, 1 \mathrm{H},=\mathrm{NH})$. MS: $m / z 383$ $\left([\mathrm{M}+2]^{+}, 30 \%\right)$. Calcd for $\mathrm{C}_{24} \mathrm{H}_{19} \mathrm{~N}_{3} \mathrm{O}_{2}$ (381.43): C 75.57, H 5.02, N 11.02\%. Found: C 74.01, H 4.20, N 10.66\%.

Preparation of 4,5-dihydro-4-(2-imino- $2 \mathrm{H}$-chromen3-yl)-6-phenyl-4-(phenyl-amino) pyrimi-din-2(1-H)-one, (28a), and its derivative (28b).

General procedure: A mixture of equimolar amounts of $24 \mathrm{a}(3.66 \mathrm{~g}, 0.01 \mathrm{~mol})$ and urea $(0.60 \mathrm{~g}, 0.01 \mathrm{~mol})$ or thiourea $(0.76 \mathrm{~g}, 0.01 \mathrm{~mol})$ and $0.1 \mathrm{~mL}$ piperidine was refluxed for $8 \mathrm{~h}$. in $30 \mathrm{~mL}$ of ethanol. The solvent was evaporated under vacuum, and the residue was poured to $30 \mathrm{ml}$ acidified cold water and then triturated with methanol. The product were filtered and crystallized from etha- nol.

Compound 28a. Brown crystals (Ethanol); yield 67\%, m.p. $196^{\circ} \mathrm{C}-198^{\circ} \mathrm{C}$. IR: 1685 - $1705(\mathrm{C}=\mathrm{O}), 3063-3288$ (NH). 1H NMR (DMSO): 2.9 (s, 2H, CH2), $4.1(s, 1 \mathrm{H}$, NH-amine), 7.2 - 7.8 (m, 15H, Ar-H), 8.1 ( $s, \mathrm{H}, \mathrm{NH}$-amide) and $11.5(\mathrm{~s}, 1 \mathrm{H},=\mathrm{NH}), \mathrm{MS}: \mathrm{m} / \mathrm{z} 408\left([\mathrm{M}]^{+}, 45 \%\right)$. Calcd for $\mathrm{C} 25 \mathrm{H} 20 \mathrm{~N} 4 \mathrm{O} 2$ (408.45): C 73.51, H 4.94, N 13.72\%. Found: C 72.01, H 3.20, N 12.66\%.

Compound 28b. Brown crystals (Methanol), yield 59\%, m.p. $210^{\circ} \mathrm{C}-112^{\circ} \mathrm{C}$. IR: $1230(\mathrm{C}=\mathrm{S}), 3063-3288$ (NH), MS: $m / z 422$ ([M-2] $]^{+}, 35 \%$ ). Calcd for $\mathrm{C}_{25} \mathrm{H}_{20} \mathrm{~N}_{4} \mathrm{OS}$ (424.52): C 70.73, H 4.75, N 13.20\%. Found: C 69.01, H 3.20 , N $12.66 \%$.

Preparation of 1, 2, 5, 6-tetrahydro-6-(2-imino-2Hchromen-3-yl)-2-oxo-4-phenyl-6-(phenylamino) pyrinecarbonitrile, (29a) and its derivatives (29b - 29e) and (30).

General procedure: A mixture of 24a (3.66 g, 0.01 $\mathrm{mol})$ and cyanoacetamide $(0.84 \mathrm{~g}, 0.01 \mathrm{~mol})$ in $30 \mathrm{ml}$ of ethanol in the presence of $0.1 \mathrm{ml}$ of piperidine was refluxed for $8 \mathrm{~h}$. The reaction mixture was concentrated under vacuum and the residue washed with acidified cold water and then triturated with methanol. The solid product formed was filtered and crystallized from ethanol to afford 29a in 65\% yield. In analogously, the Chalcone 24a was reacted with cyanothioacetamide $(1.0 \mathrm{~g}, 0.01 \mathrm{~mol}), 2$ cyano- $N$ - $p$-tolylacetamide $(1.74 \mathrm{~g}, 0.01 \mathrm{~mol}), 2$-cyanoacetohydrazide $(0.99 \mathrm{~g}, 0.01 \mathrm{~mol}), 2$-cyano- $N$-phenylacetamide (1.6 g, $0.01 \mathrm{~mol})$ and 2-chloroacetamide $(0.93 \mathrm{~g}$, $0.01 \mathrm{~mol}$ ) to yield the pyridine derivatives $29 \mathbf{b}-\mathbf{2 9 e}$ and 30 respectively.

Compound 29a. Yellow crystals (Methanol), yield 56\%, m.p. $184^{\circ} \mathrm{C}-186^{\circ} \mathrm{C}$. IR: $1689-1705(\mathrm{C}=\mathrm{O}), 2220(\mathrm{CN})$, 3188 - 3244(NH). ${ }^{1} \mathrm{H}$ NMR (DMSO): $2.9\left(s, 2 \mathrm{H}, \mathrm{CH}_{2}\right)$, 4.1 ( $s, 1 \mathrm{H}, \mathrm{NH}$-amine), 7.2 - $7.8(\mathrm{~m}, 15 \mathrm{H}, \mathrm{Ar}-\mathrm{H}), 8.1(\mathrm{~s}, \mathrm{H}$, $\mathrm{NH}$-amide) and $11.5(s, 1 \mathrm{H},=\mathrm{NH}), \mathrm{MS}: m / z 432\left([\mathrm{M}]^{+}\right.$, $65 \%$ ). Calcd for $\mathrm{C}_{27} \mathrm{H}_{20} \mathrm{~N}_{4} \mathrm{O}_{2}$ (432.47). C 74.98, H 4.66, N 12.95\%. Found: C 73.01, H 3.20, N 11.66\%.

Compound 29b. Pale yellow crystals (Methanol), yield $50 \%$, m.p. $240^{\circ} \mathrm{C}-242^{\circ} \mathrm{C}$. IR: $1251(\mathrm{C}=\mathrm{S}), 2218(\mathrm{CN})$, 3188 - $3244(\mathrm{NH})$. MS: $m / z 448\left([\mathrm{M}]^{+}, 45 \%\right)$. Calcd for $\mathrm{C}_{27} \mathrm{H}_{20} \mathrm{~N}_{4} \mathrm{OS}$ (448.54). C 72.30, H 4.49, N 12.49, S 7.15\%. Found: C 71.11, H 3.21, N 11.12, S 6.55\%.

Compound 29c. Pale red crystals (Methanol), yield 60\%, m.p. $235^{\circ} \mathrm{C}-238^{\circ} \mathrm{C}$, IR: $1689-1705(\mathrm{C}=\mathrm{O}), 2210(\mathrm{CN})$, 3188 - $3244(\mathrm{NH}), \mathrm{MS}: \mathrm{m} / z 522\left([\mathrm{M}]^{+}, 40 \%\right)$. Calcd for $\mathrm{C}_{34} \mathrm{H}_{26} \mathrm{~N}_{4} \mathrm{O}_{2}$ (522.6): C 78.14, H 5.01, N 10.72\%. Found: C 77.01, H 4.20, N 9.66\%.

Compound 29d. Pale red crystals (Methanol), yield 75\%, m.p. $217^{\circ} \mathrm{C}-219^{\circ} \mathrm{C}$. IR: $1689-1705(\mathrm{C}=\mathrm{O}), 2210(\mathrm{CN})$, 3188 - $3344\left(\mathrm{NH}\right.$ and $\left.\mathrm{NH}_{2}\right)$. MS: $m / z 447\left([\mathrm{M}]^{+}, 35 \%\right)$. Calcd for $\mathrm{C}_{27} \mathrm{H}_{21} \mathrm{~N}_{5} \mathrm{O}_{2}$ (447.49): $\mathrm{C} 71.47, \mathrm{H} 4.73, \mathrm{~N}$ 15.65\%. Found: C 70.01, H 3.20, N 14.66\%.

Compound 29e. Yellow crystals (Methanol), yield 35\%, 
m.p. $280^{\circ} \mathrm{C}-282^{\circ} \mathrm{C}$. IR: $1689-1705(\mathrm{C}=\mathrm{O}), 2210(\mathrm{CN})$, 3188 - 3344 ( $\mathrm{NH}$ and $\left.\mathrm{NH}_{2}\right) .{ }^{1} \mathrm{H}$ NMR (DMSO): $1.9(s, 2 \mathrm{H}$, $\left.\mathrm{CH}_{2}\right), 2.1\left(s, 2 \mathrm{H}, \mathrm{NH}_{2}\right), 4.1(s, 1 \mathrm{H},-\mathrm{NHPh}), 7.2$ - $7.8(\mathrm{~m}$, $20 \mathrm{H}, \mathrm{Ar}-\mathrm{H})$ and $11.5(s, 1 \mathrm{H},=\mathrm{NH}), \mathrm{MS}: m / z 510[\mathrm{M}+2]^{+}$, $50 \%$ ). Calcd for $\mathrm{C}_{33} \mathrm{H}_{24} \mathrm{~N}_{4} \mathrm{O}_{2}$ (508.57). C 77.93, H 4.76, N 11.02\%. Found: C 76.41, H 3.10, N 10.06\%.

Compound 30. Brown crystals (Methanol), yield 50\%, m.p. $220^{\circ} \mathrm{C}-222^{\circ} \mathrm{C}$. IR: $1699-1705(\mathrm{C}=\mathrm{O}), 3188-3244$ (NH). ${ }^{1} \mathrm{H}$ NMR (DMSO): $1.9\left(s, 2 \mathrm{H}, \mathrm{CH}_{2}\right), 4.1(s, 1 \mathrm{H}, \mathrm{NH}-$ amine), 7.2 - $7.8(m, 15 \mathrm{H}, \mathrm{Ar}-\mathrm{H}), 8.1(\mathrm{~s}, \mathrm{H}, \mathrm{NH})$ and $11.5(s$, $1 \mathrm{H},=\mathrm{NH})$, MS: $m / z \quad 441\left([\mathrm{M}]^{+}, 55 \%\right)$. Calcd for $\mathrm{C}_{26} \mathrm{H}_{20} \mathrm{~N}_{3} \mathrm{O}_{2} \mathrm{Cl}$ (441.91). C 70.67, H 4.56, N 9.51, Cl 8.02\%. Found: C 69.11, H 3.20, N 8.26, Cl 7.61\%.

Preparation of 2-(2-imino-2H-chromen-3-yl)-4-phenyl-2-(phenylamino)pyrido-[1,2-a]benzim-idazo-lo-5carbonitriles, (31). A mixture of 24a (3.66 g, $0.01 \mathrm{~mol})$, 2-cyanomethylbenzimidazole $(1.57 \mathrm{~g}, 0.01 \mathrm{~mol})$ and 0.1 $\mathrm{mL}$ of piperidine in $30 \mathrm{~mL}$ of ethanol was refluxed for $8 \mathrm{~h}$. The solvent was evaporated under vacuum and the residue was washed by acidified cold water and then triturated with methanol. The solid product was filtered and crystallized from ethanol, as brown crystals, yield 65\%, m.p. $278^{\circ} \mathrm{C}-280^{\circ} \mathrm{C}$. IR: $2220(\mathrm{CN}), 3188-3244(\mathrm{NH}) .{ }^{1} \mathrm{H}$ NMR $\left(\mathrm{CDCl}_{3}\right): 2.9\left(s, 2 \mathrm{H}, \mathrm{CH}_{2}\right), 4.1(s, 1 \mathrm{H}, \mathrm{NH}$-amine), 6.9 - $7.6(m, 19 \mathrm{H}, \mathrm{Ar}-\mathrm{H})$ and $11.5(s, 1 \mathrm{H},=\mathrm{NH}), \mathrm{MS}: m / z$ $505\left([\mathrm{M}]^{+}, 35 \%\right)$. Calcd for $\mathrm{C}_{33} \mathrm{H}_{23} \mathrm{~N}_{5} \mathrm{O}$ (505.57): C 78.40, H 4.59, N 13.85\%. Found: C 77.01, H 3.20, N 12.66\%.

Preparation of 2,3-dihydro-2-(2-imino-2H-chrom-en-3-yl)- $N$,4-diphenyl-benzo[ $b][1,4]$ oxazepin-2-amine, (32a) and its derivatives, (32b).

General procedure: A mixture of an equimolar amounts of 24a (3.66 g, $0.01 \mathrm{~mol})$ and $(1.09 \mathrm{~g}, 0.01 \mathrm{~mol}) o-$ aminophenol and $(1.25 \mathrm{~g}, 0.01 \mathrm{~mol}) o$-aminothiophenol respectively, in $30 \mathrm{~mL}$ ethanol containing $0.1 \mathrm{~mL}$ of piperidine was refluxed for $8 \mathrm{hr}$. The reaction mixture was concentrated, cooled then poured into ice $/ \mathrm{H}_{2} \mathrm{O}$ mixture. The solid product thus so formed was filtered, washed for several times with water and crystallized from ethanol.

Compound 32a. Brown crystals (Ethanol), yield 60\%, m.p. $282^{\circ} \mathrm{C}-284^{\circ} \mathrm{C}$, IR: $3289(\mathrm{NH}),{ }^{1} \mathrm{H}$ NMR (DMSO): 2.9( $\left.s, 2 \mathrm{H}, \mathrm{CH}_{2}\right), 4.1(s, 1 \mathrm{H}, \mathrm{NH}$-amine), 6.7 - $7.3(\mathrm{~m}, 19 \mathrm{H}$, Ar-H) and 11.5(s, 1H, = NH), MS: $m / z 457\left([\mathrm{M}]^{+}, 55 \%\right)$. Calcd for $\mathrm{C}_{30} \mathrm{H}_{23} \mathrm{~N}_{3} \mathrm{O}_{2}$ (457.52): C 78.75, H 5.07, N 9.18\%. Found: C 77.01, H 4.20, N 8.66\%.

Compound 32b. Yellow crystals (Ethanol), yield $45 \%$, m.p. $270^{\circ} \mathrm{C}-272^{\circ} \mathrm{C}$. IR: $3188-3244(\mathrm{NH}),{ }^{1} \mathrm{H} \mathrm{NMR}\left(\mathrm{CDCl}_{3}\right)$ : $1.9\left(s, 2 \mathrm{H}, \mathrm{CH}_{2}\right), 4.1$ ( $s, 1 \mathrm{H}, \mathrm{NH}$-amine), 6.9 - $7.6(m, 19 \mathrm{H}$, $\mathrm{Ar}-\mathrm{H})$ and $11.5(s, 1 \mathrm{H},=\mathrm{NH}), \mathrm{MS}: m / z 476\left([\mathrm{M}+3]^{+}, 45 \%\right)$. Calcd for $\mathrm{C}_{30} \mathrm{H}_{23} \mathrm{~N}_{3} \mathrm{OS}$ (473.59). C 76.08, H 4.90, N 8.87, S 6.77\%. Found: C 77.01, H 3.20, N 12.66, S 5.12\%.

Preparation of 2-(dicyanomethylene)-1,2,5,6-tetrahydro-6-(2-imino-2H-chromen-3-yl)-4-phenyl-6-(phenylamino)pyridine-3-carbonitrile, (33a) and its derivatives (33b).
General procedure: A mixture of $24 a(0.366 \mathrm{~g}, 0.001$ $\mathrm{mol})$ and 2-aminoprop-1-ene-1,1,3-tricarbonitrile (0.132 $\mathrm{g}, 0.001 \mathrm{~mol}$ ) in $30 \mathrm{~mL}$ of ethanol in the presence of 0.1 $\mathrm{mL}$ of piperidine was refluxed for $8 \mathrm{~h}$. The reaction mixture was concentrated under vacuum and the residue washed with acidified cold water and then triturated with methanol. The solid product formed was filtered and crystallized from methanol to afford 33a in $60 \%$ yield. In analogously, the Chalcone 34a was reacted with ethyl 3-amino-2, 4-dicyanobut-2-enoate $(1.79 \mathrm{~g}, 0.01 \mathrm{~mol})$ to yield the pyridine derivative $33 \mathbf{b}$.

Compound 33a. Yellow crystals (Methanol), yield 60\%, m.p. $184^{\circ} \mathrm{C}-186^{\circ} \mathrm{C}$. IR: $2219(\mathrm{CN}), 3289(\mathrm{NH}) .{ }^{1} \mathrm{H}$ NMR (DMSO): $2.9\left(s, 2 \mathrm{H}, \mathrm{CH}_{2}\right), 4.1(s, 1 \mathrm{H}, \mathrm{NH}$-amine), 6.7 $7.3(\mathrm{~m}, 15 \mathrm{H}, \mathrm{Ar}-\mathrm{H}), 8.1(\mathrm{~s}, \mathrm{H}, \mathrm{NH})$ and $11.5(s, 1 \mathrm{H},=\mathrm{NH})$, MS: $m / z 482\left([\mathrm{M}+2]^{+}, 35 \%\right)$. Calcd for $\mathrm{C}_{30} \mathrm{H}_{20} \mathrm{~N}_{6} \mathrm{O}$ (480.52). C 74.99, H 4.20, N 17.49\%. Found: C 73.01, H $3.20, \mathrm{~N} 16.66 \%$.

Compound 33b. Pale yellow crystals (Methanol), yield $45 \%$, m.p. $240^{\circ} \mathrm{C}-242^{\circ} \mathrm{C}$. IR: $2219(\mathrm{CN}), 3289(\mathrm{NH}) .{ }^{1} \mathrm{H}$ NMR (DMSO): $\delta 1.2\left(t, 3 \mathrm{H}, \mathrm{CH}_{3}\right), 2.2\left(s, 2 \mathrm{H}, \mathrm{CH}_{2}\right), 3.7(q$, $\left.2 \mathrm{H}, \mathrm{CH}_{2}\right), 4.1$ ( $s, 1 \mathrm{H}, \mathrm{NH}$-amine), 6.7 - 7.9 ( $\left.m, 15 \mathrm{H}, \mathrm{Ar}-\mathrm{H}\right)$ and $11.5(s, 1 \mathrm{H},=\mathrm{NH}), \mathrm{MS}: \mathrm{m} / z 527\left([\mathrm{M}]^{+}, 35 \%\right)$. Calcd for $\mathrm{C}_{32} \mathrm{H}_{25} \mathrm{~N}_{5} \mathrm{O}_{3}$ (527.57). Calcd: C 72.85, H 4.78, N 13.27\%. Found: C 71.14, H 3.01, N 12.88\%.

Preparation of 3-chloro-4- (2-imino-2H-chromen-3yl)-1-phenyl-4-(phenyl-amino) azetidin-2-one, (34a) and its derivatives, $(34 b-34 e)$.

Compound 34a. Brown crystals (Methanol) yield 60\%, m.p. $280^{\circ} \mathrm{C}-282^{\circ} \mathrm{C}$. IR: $1705(\mathrm{C}=\mathrm{O}), 3289(\mathrm{NH}) .{ }^{1} \mathrm{H}$ NMR (DMSO): 4.1 ( $s, 1 \mathrm{H}, \mathrm{NH}$-amine), $5.2(s, 1 \mathrm{H}, \mathrm{CH}$, $\mathrm{C}$-3 of $\beta$-lactam ring), $7.1-7.6(\mathrm{~m}, 15 \mathrm{H}, \mathrm{Ar}-\mathrm{H})$ and $11.5(\mathrm{~s}$, $1 \mathrm{H},=\mathrm{NH}), \mathrm{MS}: \mathrm{m} / z \quad 415\left([\mathrm{M}]^{+}, 40 \%\right)$, Calcd for $\mathrm{C}_{24} \mathrm{H}_{18} \mathrm{~N}_{3} \mathrm{O}_{2} \mathrm{Cl}$ (415.87): $\mathrm{C}$ 69.31, $\mathrm{H} 4.36, \mathrm{~N} 10.10, \mathrm{Cl}$ 8.5279\%. Found: C 68.19, H 3.21, N 9.18, Cl 7.12\%.

Compound 34b. Pale yellow crystals (Methanol), yield $63 \%$, m.p. $275^{\circ} \mathrm{C}-277^{\circ} \mathrm{C}$. IR: $1705(\mathrm{C}=\mathrm{O}), 3289(\mathrm{NH})$, 3388(OH). ${ }^{1} \mathrm{H}$ NMR (DMSO): 4.1 ( $s, 1 \mathrm{H}, \mathrm{NH}$-amine), 5.2 ( $s, 1 \mathrm{H}, \mathrm{CH}, \mathrm{C}-3$ of $\beta$-lactam ring), $7.1-7.6(m, 14 \mathrm{H}, \mathrm{Ar}-\mathrm{H})$, $11.5(s, 1 \mathrm{H},=\mathrm{NH})$ and $10.1(s, 1 \mathrm{H}$, phenolic $\mathrm{OH})$. MS: $m / z$ 431([M] $\left.]^{+}, 55 \%\right)$, Calcd for $\mathrm{C}_{24} \mathrm{H}_{18} \mathrm{~N}_{3} \mathrm{O}_{3} \mathrm{Cl}$ (431.87): $\mathrm{C}$ 66.75, H 4.20, N 9.73, Cl 8.21\%. Found: C 65.09, H 3.52, $\mathrm{N} \mathrm{9.08,} \mathrm{Cl} 7.12 \%$.

Compound 34c. Pale brown crystals (Methanol), yield $70 \%$, m.p. $210^{\circ} \mathrm{C}-212^{\circ} \mathrm{C}$. IR: $1705(\mathrm{C}=\mathrm{O}), 3289(\mathrm{NH}) .{ }^{1} \mathrm{H}$ NMR $\left(\mathrm{CDCl}_{3}\right): 4.1(s, 1 \mathrm{H}, \mathrm{NH}$-amine $), 5.2(s, 1 \mathrm{H}, \mathrm{CH}$, $\mathrm{C}$-3 of $\beta$-lactam ring), $7.1-7.6(\mathrm{~m}, 14 \mathrm{H}, \mathrm{Ar}-\mathrm{H})$ and $11.5(\mathrm{~s}$, $1 \mathrm{H},=\mathrm{NH})$. MS: $m / z 460\left([\mathrm{M}]^{+}, 40 \%\right)$, Calcd for $\mathrm{C}_{24} \mathrm{H}_{17} \mathrm{~N}_{4} \mathrm{O}_{4} \mathrm{Cl}$ (460.87): C 62.55, H 3.72, N 12.16, Cl 7.69\%. Found: C 61.07, H 2.22, N 11.18, Cl 6.11\%.

Compound 34d. Brown crystals (Ethanol), yield 62\%, m.p. $175^{\circ} \mathrm{C}-177^{\circ} \mathrm{C}$. IR: $1705(\mathrm{C}=\mathrm{O}), 3289(\mathrm{NH})$. MS: $\mathrm{m} / \mathrm{z}$ $461\left([\mathrm{M}+1]^{+}, 55 \%\right)$, Calcd for $\mathrm{C}_{24} \mathrm{H}_{17} \mathrm{~N}_{4} \mathrm{O}_{4} \mathrm{Cl}$ (460.87): $\mathrm{C}$ 62.55, H 3.72, N 12.16, Cl 7.69\%. Found: C 61.17, H 2.25, 
N 11.48, Cl 6.21\%.

Compound 34e. Yellow crystals (Ethanol), yield 55\%, m.p. $250^{\circ} \mathrm{C}-252^{\circ} \mathrm{C}$. IR: $1705(\mathrm{C}=\mathrm{O}), 3289(\mathrm{NH}) .{ }^{1} \mathrm{H}$ $\mathrm{NMR}\left(\mathrm{CDCl}_{3}\right): 4.1(s, 1 \mathrm{H}, \mathrm{NH}$-amine $), 5.2(s, 1 \mathrm{H}, \mathrm{CH}, \mathrm{C}-3$ of $\beta$-lactam ring), $7.1-7.6(m, 14 \mathrm{H}, \mathrm{Ar}-\mathrm{H})$ and $11.5(s, 1 \mathrm{H}$, $=\mathrm{NH})$. MS: $m / z 450\left([\mathrm{M}]^{+}, 65 \%\right)$, Calcd for $\mathrm{C}_{24} \mathrm{H}_{17} \mathrm{~N}_{3} \mathrm{O}_{2} \mathrm{Cl}_{2}$ (450.32): C 64.01, H 3.81, N 9.33, Cl 7.11\%. Found: C 63.07, H 2.82, N 8.18, Cl 6.41\%.

Preparation of 2-(2-imino-2H- chromen-3-yl)-3-phenyl-2-(phenylamino) thiazolidin-4-one, (35a) and its derivatives (35b - 35e).

General procedure: An equimolar mixture of 25a ( 0.99 g, $0.003 \mathrm{~mol})$ and thioglycolic acid $(0.276 \mathrm{~mL}, 0.003 \mathrm{~mol})$ in dry benzene $(20 \mathrm{~mL})$ was refluxed for $10 \mathrm{~h}$. The reaction mixture was evaporated to dryness under reduced pressure. The thiazolidinone was separated off, washed with ether and crystallized from ethanol. Analogously, 25b - 25e reacted with thioglycolic acid to yield 35b - 35e.

Compound 35a. Yellow crystals (ethanol), yield 60\%, m.p. $185^{\circ} \mathrm{C}-187^{\circ} \mathrm{C}$. IR: 1230 (CS), $1695(\mathrm{C}=\mathrm{O}), 3289$ (NH). ${ }^{1} \mathrm{H}$ NMR (DMSO): $3.8\left(s, 2 \mathrm{H}, \mathrm{CH}_{2}\right), 4.1(s, 1 \mathrm{H}, \mathrm{NH}-$ amine), $6.8-7.8(\mathrm{~m}, 15 \mathrm{H}, \mathrm{Ar}-\mathrm{H})$ and $11.5(s, 1 \mathrm{H},=\mathrm{NH})$. MS: $m / z 411\left([\mathrm{M}-2]^{+}, 35 \%\right)$. Calcd for $\mathrm{C}_{24} \mathrm{H}_{19} \mathrm{~N}_{3} \mathrm{O}_{2} \mathrm{~S}$ (413.49): C 69.71, H 4.63, N 10.16, S 7.91\%. Found: C 68.46, H 3.17, N 9.46, S 6.55\%.

Compound 35b. Yellow crystals (Methanol), yield 70\%, m.p. $215^{\circ} \mathrm{C}-117^{\circ} \mathrm{C}$. IR: 1230 (CS), $1695(\mathrm{C}=\mathrm{O}), 3289$ (NH). ${ }^{1} \mathrm{H}$ NMR (DMSO): $3.8\left(s, 2 \mathrm{H}, \mathrm{CH}_{2}\right), 4.1(s, 1 \mathrm{H}, \mathrm{NH}-$ amine), 6.8 - $7.8(m, 15 \mathrm{H}, \mathrm{Ar}-\mathrm{H}), 11.5(\mathrm{~s}, 1 \mathrm{H},=\mathrm{NH})$ and $10.1\left(s, 1 \mathrm{H}\right.$, phenolic OH). MS: $m / z 429\left([\mathrm{M}]^{+}, 44 \%\right)$. Calcd for $\mathrm{C}_{24} \mathrm{H}_{19} \mathrm{~N}_{3} \mathrm{O}_{3} \mathrm{~S}$ (429.49): C 67.12, H 4.46, N 9.78, $\mathrm{S}$ 7.47\%. Found: C 66.46, H 3.17, N 8.46, S 6.25\%.

Compound 35c. Pale brown crystals (Ethanol), yield $76 \%$, m.p. $198^{\circ} \mathrm{C}-200^{\circ} \mathrm{C}$. IR: 1230 (CS), $1695(\mathrm{C}=\mathrm{O})$, $3289(\mathrm{NH}) .{ }^{1} \mathrm{H}$ NMR (DMSO): $3.8\left(s, 2 \mathrm{H}, \mathrm{CH}_{2}\right), 4.1(s$, $1 \mathrm{H}, \mathrm{NH}$-amine $), 6.8-7.8(m, 14 \mathrm{H}, \mathrm{Ar}-\mathrm{H})$ and $11.5(s, 1 \mathrm{H}$, $=\mathrm{NH})$. MS: $m / z 457$ ([M-1] $]^{+}, 55 \%$ ). Calcd for $\mathrm{C}_{24} \mathrm{H}_{18} \mathrm{~N}_{40} \mathrm{O}$ S (458.49): C 62.87, H 3.96, N 12.22, S 6.99\%. Found: C 61.66, H 3.17, N 11.16, S 5.55\%.

Compound 35d. Yellow crystals (Ethanol), yield 65\%, m.p. $290^{\circ} \mathrm{C}-292^{\circ} \mathrm{C}$. IR: 1230 (CS), 1695 (C=O), 3289 (NH). MS: $m / z 458\left([\mathrm{M}]^{+}, 55 \%\right)$. Calcd for $\mathrm{C}_{24} \mathrm{H}_{18} \mathrm{~N}_{40} \mathrm{O}_{4} \mathrm{~S}$ (458.49): C 62.87, H 3.96, N 12.22, S 6.99\%. Found: C 61.12, H 3.74, N 11.18, S 6.25\%.

Compound 35e. Brown crystals (Methanol), yield 62\%, m.p. $235^{\circ} \mathrm{C}-237^{\circ} \mathrm{C}$. IR: 1230 (CS), 1695 (C=O), 3289 (NH). ${ }^{1} \mathrm{H}$ NMR (DMSO): $3.8\left(s, 2 \mathrm{H}, \mathrm{CH}_{2}\right), 4.1(s, 1 \mathrm{H}$, NH-amine), $6.8-7.8(m, 14 \mathrm{H}, \mathrm{Ar}-\mathrm{H})$ and $11.5(s, 1 \mathrm{H},=\mathrm{NH})$. MS: $m / z 447\left([\mathrm{M}]^{+}, 65 \%\right)$. Calcd for $\mathrm{C}_{24} \mathrm{H}_{18} \mathrm{~N}_{3} \mathrm{O}_{2} \mathrm{SCl}$ (447.94): C 64.35, H 4.05, N 9.38, S 7.91\%. Found: C 63.63, H 3.57, N 9.16, S 6.55\%.

\section{Results and Discussion}

The synthetic procedures adopted to obtain the target compounds are depicted in Schemes 1 - 10. The starting compound, 2-imino- $\mathrm{N}$-phenyl-2H-chromene-3-carboxamide $\mathbf{1}$, was prepared according to the previously reported procedure [18]. Thus, refluxing of compound 1 with triethyl orthoformate in dimethylformamide affording 2-ethoxy-2,3dihydro-3-phenylchromeno[2,3- $d$ ] pyrimidin-4-one 2, (Scheme 1). The structure of the later product was based on IR, ${ }^{1} \mathrm{H}$ NMR, and mass spectra. The IR spectrum of $\mathbf{2}$ showed the lack of any absorption of the NH functions, and the ${ }^{1} \mathrm{H}$ NMR spectrum $\left(\mathrm{CDCl}_{3}\right)$ displayed a triplet at $\delta 1.2$ and a quartet at $3.9 \mathrm{ppm}$ due to the ethoxy protons and a multiplet at $6.7-7.9 \mathrm{ppm}$ due to aromatic protons, respectively. The MS of 2 showed the $[\mathrm{M}]^{+}$ion at $m / z 320$ (65\%). Furthermore, compound 2 was allowed to react with hydrazine hydrate in ethanolic solution using piperidine as a catalyst yielding 2-hydrazinyl-2, 3-dihydro-3-phenyl-chromeno[2,3d] pyrimidin-4-one, $\mathbf{3}$. The structure of $\mathbf{3}$ was confirmed based on elemental and spectroscopic analysis. The IR spectrum of $\mathbf{3}$ showed the presence of absorption bands at v 1670,3282 and $3432 \mathrm{~cm}^{-1}$ due to $\mathrm{CO}, \mathrm{NH}$ and $\mathrm{NH}_{2}$ groups, respectively. The ${ }^{1} \mathrm{H}$ NMR spectrum (DMSO) displayed three signals at $\delta 4.82,5.1$ and 8.85 due to $\mathrm{NH}_{2}$, pyrimidine proton and $\mathrm{NH}$ respectively, and a multiplet at $6.7-7.9 \mathrm{ppm}$, for aromatic protons. While, the MS of 3 showed $\mathrm{m} / \mathrm{z}$ at $309\left([\mathrm{M}+3]^{+}, 45 \%\right)$.

Compound 3 was utilized as a key intermediate for the synthesis of some new pyrazole derivatives based on mild and efficient reaction of compound $\mathbf{3}$ with some of laboratory available compounds. Thus, Compound 3 reacted with ethyl 3-amino-2,4-dicyanobut-2-enoate in boiling DMF containing a catalytic amount of piperidine. Two isomeric products seemed possible for this reaction $\mathbf{5}$ or $\mathbf{6}$, (Scheme 2). Structure 6 ruled out based on analytical and spectroscopic data (IR, ${ }^{1} \mathrm{H}$ NMR and MS). Thus, the IR spectra of the product 5 showed the presence of absorption bands at $v 1699,2219$ and $3422 \mathrm{~cm}^{-1}$ due to $\mathrm{C}=\mathrm{O}, \mathrm{CN}$ and $\mathrm{NH}_{2}$ groups, respectively. Accordingly, the ${ }^{1} \mathrm{H}$ NMR spectrum (DMSO) of the product 5 showed three singlet signals at $\delta 2.9,3.5$ and 3.7 due to the methylene and two $\mathrm{NH}_{2}$ groups respectively, and a multiplet at $6.7-7.9 \mathrm{ppm}$, due to aromatic protons. The MS of 5 displayed $[\mathrm{M}]^{+}$at $\mathrm{m} / \mathrm{z} 439(60 \%)$. It is note worth that, trial to cyclise 5 in boiling pyridine was unsuccessful, (Scheme 2).

Also, compound 3 reacted with diethyl malonate in boiling DMF containing a catalytic amount of piperidine afforded 1-(3,4-dihydro-4-oxo-3-phenyl-2H-chromeno[2, 3-d]pyrimidin-2-yl)pyrazolidine-3,5-dione 7, (Scheme 3). The structure of 7 was confirmed based on elemental and

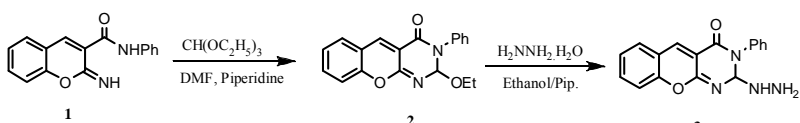

Schemes 1. Synthetic route to variously substituted 2, 3dihydro-3-phenylchromeno[2,3- $d$ ] pyrimidin-4-one. 
spectroscopic analysis. The IR spectra 7 showed the presence of absorption band centered between $v 1685$ - 1705, $3212 \mathrm{~cm}^{-1}$ due to $\mathrm{CO}$ and $\mathrm{NH}$ functions respectively. Accordingly, the ${ }^{1} \mathrm{H}$ NMR spectrum $\left(\mathrm{CDCl}_{3}\right)$ of 7 showed three singlet signals at $\delta 3.2,5.8$ and $8.1 \mathrm{ppm}$ due to the methylene, methine and $\mathrm{NH}$ pyrazole ring protons respectively, and a multiplet at $\delta 6.1-7.8$ for aromatic protons. The MS of 7 displayed $m / z$ at $374\left([\mathrm{M}]^{+}, 55 \%\right)$.

Similarly, compound $\mathbf{3}$ was allowed to react with ethyl 2-cyanoacetate, ethyl 3-oxobutanoate and pentane-2,4-dione to afford the corresponding pyrazole derivatives 8 - 10 respectively, (Schemes 3).

Likewise, compound 3 was reacted with 2-(ethoxymethylene) malononitrile and 2-cyano- $N$-phenylacetamide gave the corresponding pyrazole derivatives 12, 13a, b respectively, (Scheme 4). While the reaction of compound 3 with nitrous acid afforded the tetrazolo[1,5a]pyrimidine derivatives 11, (Scheme 4). The structure of these compounds was in agreement with analytical and spectroscopic data. The IR spectrum of $\mathbf{1 1}$ showed the presence of absorption bands at $v 1699 \mathrm{~cm}^{-1}$ due to CO group. While, the MS of compound 11 showed $\mathrm{m} / \mathrm{z}$ at $317\left([\mathrm{M}]^{+}, 60 \%\right)$, ( Scheme 4).

On the other hand, the hydrazine 3 reacted with aromatic aldehydes afforded 2-(2-benzylidenehydrazinyl)-3phenyl-3H-chromeno[2,3- $d]$ pyrimidin-4-one 14, which was cyclized to 5-oxo-1-(4-oxo-3-phenyl-4,10-dihydro$3 H$-chromeno[2,3- $d$ ]pyrimi-din-2-yl)-3-phenylpyrazolidine-4-carbonitrile 16 by treatment with ethyl cyanoacetate. It seemed that the addition of active methylene hydrogen of ethyl cyanoacetate to the imino carbon of $\mathbf{1 4}$ gave the intermediate $\mathbf{1 5}$, which subsequently cyclized via elimination of ethanol molecule yielding 16. The MS of 16 showed $\mathrm{m} / \mathrm{z}$ at $462(\mathrm{M}+1)^{+}, 55 \%$. Its IR spectrum revealed absorption bands at $v 3432 \mathrm{~cm}^{-1}(\mathrm{NH}), 2217 \mathrm{~cm}^{-1}$ (CN) and $1670 \mathrm{~cm}^{-1}(\mathrm{CO})$. The ${ }^{1} \mathrm{H}$ NMR spectrum showed a multiplet signals at $\delta 6.71-7.92$ for aromatic protons and three singlet signals at $\delta 3.98,4.12$ and 4.21 due to $-\mathrm{NH}$, the two methine protons of pyrazole ring, respectively, (Scheme 5). The compound $\mathbf{1 6}$ was transformed in to the chloro derivatives $\mathbf{1 7}$, by treatment with phosphoryl chloride (Scheme 5). The MS of 17 showed at $\mathrm{m} / \mathrm{z}$ at 478 ([M $\left.-1]^{+}, 16 \%\right)$. The IR spectrum of 17 showed the presence of absorption band at $v 1700 \mathrm{~cm}^{-1}$ due to $\mathrm{C}=\mathrm{O}, 2217 \mathrm{~cm}^{-1}$ due to $\mathrm{CN}$ and $3425 \mathrm{~cm}^{-1}$ due to $\mathrm{NH}$ function. The ${ }^{1} \mathrm{HNMR}$ of compound 17 showed singlet signals at $\delta$ ppm 3.98 and 4.61 due to $\mathrm{NH}$ and methane proton of pyrazole ring.

Compound 17 was utilized as a useful starting material for the synthesis of a variety heterocycle-isolated coumarin derivatives based on mild and efficient reaction of compound 17 with some of laboratory available compounds. Thus, compound 17 reacted with 5-amine- $1 H$-1,2,4-triazole in boiling DMF containing a catalytic amount of piperidine afforded 2-(4-amino-3-phenyl-2,3-dihydro- $1 \mathrm{H}$ pyrazolo[4,3-e][1,2,4]triazolo[1,5-a]pyrimidin-1-yl)-3-phenyl-3H-chromeno[2,3- $d$ ]pyrimidin-4(10H)-one 18, (Scheme 6). Similarly, compound 17 reacted with 5-amino-3(methylthio)- $1 H$-pyrazole-4-carbonitrile yielded 4-amino7-(methylthio)-1-(4-oxo-3-phenyl-4,10-dihydro-3H-chromeno[2,3-d]pyrimidin-2-yl)-3-phenyl-2, 3-dihydro- $1 H$-dipyrazolo[1,5-a:4',3'-e]pyrimidine-6-carbonitrile 19. The

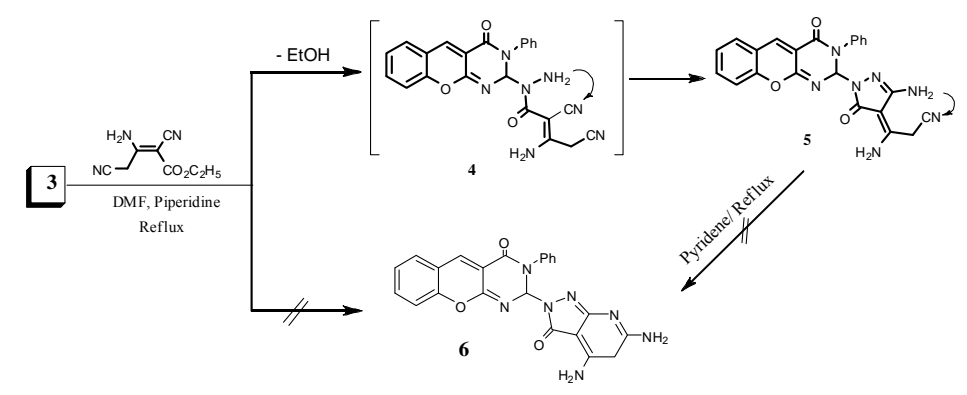

Scheme 2. Synthetic rpute to prepare isolated pyrazole derivatives and reaction conditions.

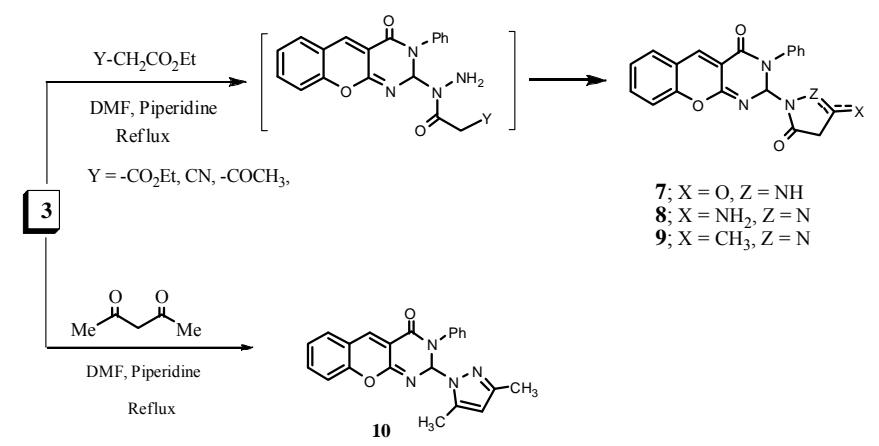

Scheme 3. Synthetic rpute to isolated oxazole 7 and pyrazole derivatives 8 - 10, and reaction conditions. 


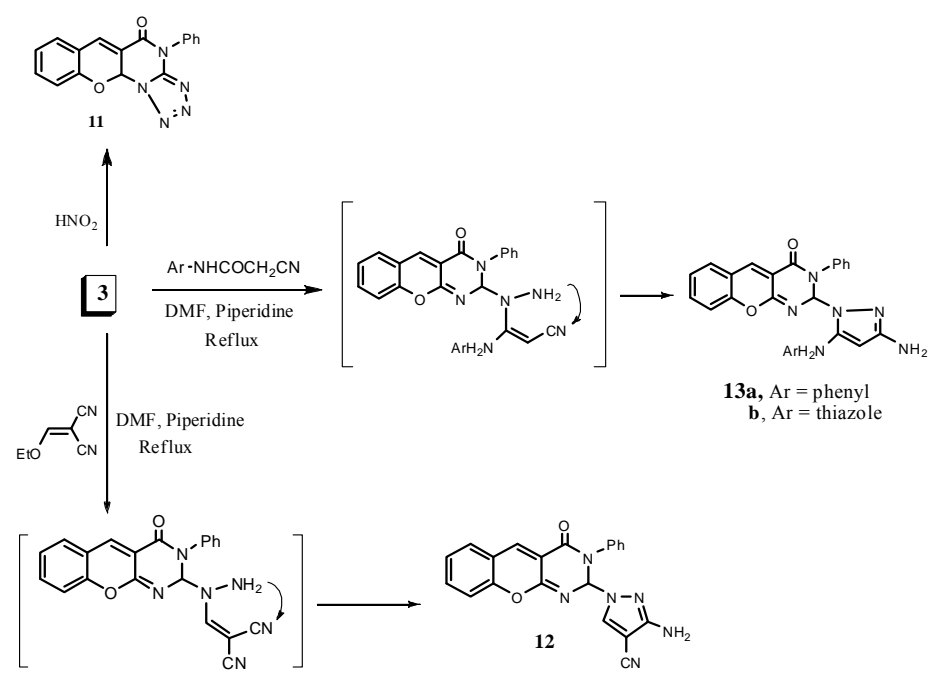

Scheme 4. Synthetic rpute to isolated pyrazole derivatives and reaction conditions.

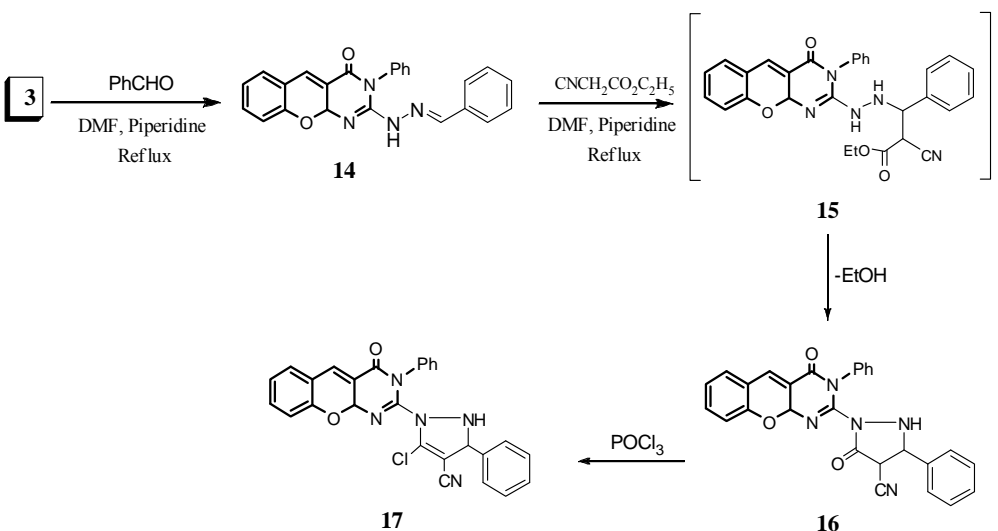

Scheme 5. Synthetic rpute to isolated pyrazole derivatives and reaction conditions.

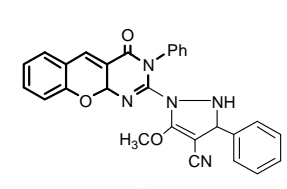

20

$$
\underset{\substack{\text { DMF, Piperidine } \\ \text { Reflux }}}{\mathrm{CH}_{3} \mathrm{ONa}} \stackrel{\substack{\text { DMF, Piperidine } \\ \text { Reflux }}}{\stackrel{\mathrm{NH}_{2}}{2}}
$$

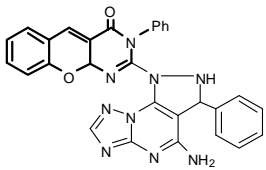

18

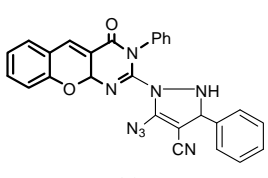

21

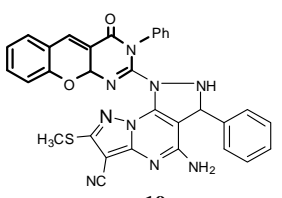

19

$$
\underset{\substack{\text { DMF, Piperidine } \\ \text { Reflux }}}{\mathrm{NaN}_{3}} \frac{\substack{\text { DMF, Piperidine } \\ \text { Reflux }}}{\stackrel{\mathrm{HC}_{3} \mathrm{~N}_{\mathrm{N}}}{\mathrm{NHH}^{-\mathrm{N}_{2}}}}
$$

$\mathrm{HN}_{2} \mathrm{NH}_{2}$ DMF, Piperidin Reflux
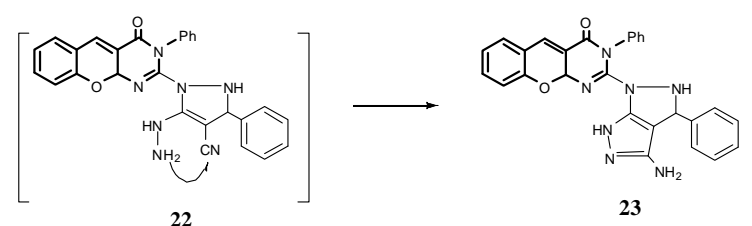

23

Scheme 6. Synthetic rpute to isolated and/or fused pyrazole derivatives 18 - 23 , and reaction conditions. 
structure of these compounds was confirmed based on elemental and spectroscopic analysis. (See experimental section). Also, the chloro derivative $\mathbf{1 7}$ was converted to the 5-methoxy-1-(4-oxo-3-phenyl-4,10-dihydro-3H-chromeno[2,3- $d]$ pyrimidin-2-yl)-3-phenyl-2,3-dihy-dro- $1 H$ pyrazole-4-carbonitrile 20 by refluxing with $\mathrm{MeONa}$, (Scheme 6). Structure 20 confirmed based on analytical and spectroscopic data (IR, ${ }^{1} \mathrm{H}$ NMR and MS). Thus, the IR spectra of the product 20 showed the presence of absorption bands at $v 1699,2219$ and $3422 \mathrm{~cm}^{-1}$ due to $\mathrm{C}=\mathrm{O}$, $\mathrm{CN}$ and $\mathrm{NH}$ groups, respectively. Accordingly, the ${ }^{1} \mathrm{H}$ NMR spectrum (DMSO) of the product 20 showed three singlet signals at $\delta 3.82,3.98$ and 4.61 due to the $-\mathrm{OCH}_{3}$, $-\mathrm{NH}$, and methine protons respectively, and a multiplet at 6.7 - $7.9 \mathrm{ppm}$, due to aromatic protons. The MS of 20 displayed [M] $]^{+}$at $m / z 475$ (62\%). We prepared 5-azido-1(4-oxo-3-phenyl-4,10-dihydro-3H-chromeno[2,3- $d$ ]pyrimidin-2-yl)-3-phenyl-2, 3-dihydro- $1 H$-pyrazole-4-carbonitrile $\mathbf{2 1}$ from the chloro compound $\mathbf{1 7}$ by reaction with $\mathrm{NaN}_{3}$ in acetone ${ }^{[21,22]}$. The structure of $\mathbf{2 1}$ was in agreement with analytical and spectroscopic data. The IR spectrum of $\mathbf{2 1}$ showed the presence of absorption bands at $v 1699 \mathrm{~cm}^{-1}$ due to $\mathrm{CO}, 2217 \mathrm{~cm}^{-1}$ due to $\mathrm{CN}$ and 3422 $\mathrm{cm}^{-1}$ due to NH groups. While, the MS of compound 21 showed $\mathrm{m} / \mathrm{z}$ at $486\left([\mathrm{M}]^{+}, 32 \%\right)$. In further reactions, chloro compound $\mathbf{1 7}$ on treatment with hydrazine hydrate in DMF containing a catalytic amount of piperidine at reflux temperature afforded 2-(4-amino-3-phenyl-2,3-dihydro-pyrazolo[3,4-c]pyrazol-1(6H)-yl)-3-phenyl-3H-chromeno[2,3- $d]$ pyrimidin-4(10H)-one 23 , The formation of 23 may be proceeded via an initial elimination of $\mathrm{HCl}$ molecule to give the intermediate 22, which cyclized by nucleophilic addition of the amino function into the cyano group yielded 23, (Scheme 6). The IR spectrum of $\mathbf{2 3}$ showed the presence of absorption bands at $v 1670 \mathrm{~cm}^{-1}$ due to $(\mathrm{CO})$ and $3432-3455 \mathrm{~cm}^{-1}$ due to $\left(\mathrm{NH}, \mathrm{NH}_{2}\right)$ with the absence of any characteristic absorption of $(\mathrm{CN})$ group. The ${ }^{1} \mathrm{H}$ NMR spectrum of $\mathbf{2 3}$ showed four singlet at $\delta 3.98$, $5.21,6.53$ and $9.21 \mathrm{ppm}$ due to the -NH amine, methine of pyrazole ring, amino and imino group respectively, and a multiplet at $\delta 6.97-7.81$ for aromatic protons. The MS of 23 displayed $\mathrm{m} / \mathrm{z}$ at $475\left(\mathrm{M}^{+}, 15 \%\right)$.

On the other hand, the carboxamide 1 easily condensed with acetophenone derivatives in DMF containing a catalytic amount of piperidine at reflux temperature to afford chromenochalcones 24a - 24e via elimination of water (Scheme 7). The IR spectrum of 24a showed absorption bands at $v 1665-1705$ and 3322 due to $\mathrm{CO}$ and $\mathrm{NH}$ groups respectively, the Ms of 24a showed $\mathrm{m} / \mathrm{z}$ at $366\left([\mathrm{M}]^{+}\right.$, $40 \%$ ). While, the ${ }^{1} \mathrm{HNMR}$ spectrum of 24a (DMSO) showed three singlet signals at $\delta 4.1,6.5$ and $11.5 \mathrm{ppm}$ due to the NH- amine, methylene and $\mathrm{NH}$-imino groups respectively, and a multiplet at $\delta 7.1-7.6$ for aromatic protons.
Likewise, compound $\mathbf{1}$ reacts with some aromatic amines to afford the corresponding Schiff's base 25a - 25e, (Scheme 7). The IR spectrum of 25a showed the presence of absorption bands at $v 3380$, due to $\mathrm{NH}$ function, with the absence of any characteristic absorption of a $\mathrm{C}=\mathrm{O}$ group. The ${ }^{1} \mathrm{H}$ NMR spectrum $\left(\mathrm{CDCl}_{3}\right)$ displayed two signals at $\delta$ 4.1, 3.7 and 11.5 due to $\mathrm{NH}$-amine, and $\mathrm{NH}$-imino groups respectively, and a multiplet at $7.1-7.6 \mathrm{ppm}$, for aromatic protons. While, the MS of 25a showed $\mathrm{m} / \mathrm{z}$ at $339\left([\mathrm{M}]^{+}\right.$, $65 \%)$.

The reactivity of exocyclic $\mathrm{C}=\mathrm{C}$ conjugated with the carbonyl group in 24a - 24e was investigated by reaction with hydrazines, hydroxylamine, urea, thiourea and some laboratory available active methylene compounds. The nature of the products obtained characterized by elemental and spectroscopic data, indicates that the reaction proceeded via condensation followed by a nucleophilic attack through $\alpha, \beta$-unsaturated ketonic group. Pyrazoles/isoxazole derivatives 26a - 26b and 27 were synthesized by treating 24a with equimolar ratios of hydrazine hydrate (or phenylhydrzine or hydroxylamine respectively) in ethanol containing a catalytic amount of piperidine, (Scheme 8). The structure of these compounds was established based on analytical and spectroscopic data. The IR spectra 26a showed the presence of absorption band centered between $v 3117-3293 \mathrm{~cm}^{-1}$ due to $\mathrm{NH}$ function, with the absence of any characteristic absorption of a $\mathrm{C}=\mathrm{O}$ group. Accordingly, the ${ }^{1} \mathrm{H}$ NMR spectrum (DMSO) of 26a showed four singlet signals at $\delta 2.9,4.1,7.1$ and $11.5 \mathrm{ppm}$ due to the proton at $\mathrm{C}-4$ of pyrazole, $\mathrm{NH}$ - amine, $\mathrm{NH}$ - hydrazide and $=\mathrm{NH}$ imino respectively, and a multiplet at $\delta 7.2-7.8$ for aromatic protons. The MS of 26a displayed $\mathrm{m} / \mathrm{z}$ at 383 $\left([\mathrm{M}+3]^{+}, 55 \%\right)$. The activation exerted by the carbonyl group on the exocyclic double bond in 24a renders them available for the cyclocondensation addition of various amino compounds such as urea and thiourea. Thus, when the chalcone 24a was reacted with an equimolar quantity of urea or thiourea respectively, (Scheme 7) an initial condensation of one amino group with the carbonyl function occurred releasing water, followed by a nucleophilic addition of the second amino group to the double bond forming 4,5-dihydro-4-(2-imino-2 $H$-chromen-3-yl)-6-phenyl-4-(phenylamino)pyrimidin-2(1H)-ones, 28a and/or thione 28b. The structures of the synthesized compounds were confirmed by analytical and spectroscopic data (IR, ${ }^{1} \mathrm{H}$ NMR and MS). The IR spectra 28a showed the presence of absorption bands centered between $v 1685$ - 1705, $3063-3288 \mathrm{~cm}^{-1}$ due to $\mathrm{C}=\mathrm{O}$ and $\mathrm{NH}$ functions, respectively. The ${ }^{1} \mathrm{H}$ NMR spectrum (DMSO) of 28a showed four singlet signals at $\delta 2.9,4.1,8.1$ and $11.5 \mathrm{ppm}$ due to the proton at $\mathrm{C}-4$ of pyrimidine, $\mathrm{NH}$-amine, $\mathrm{NH}$-amide and $\mathrm{NH}$-imino protons, respectively and a multiplet at $\delta$ 7.2 - 7.8 for aromatic protons. The MS of 28a showed a peak at $m / z 408\left([\mathrm{M}]^{+}, 45 \%\right)$. New pyridine derivatives 
have been prepared via condensation of 24a with the active methylene groups of cyanoacetamide, cyanothioacetamide, 2-cyano- $N$ - $p$-tolylacetamide, 2-cyanoacetohy-drazide and 2-cyano- $N$-phenylacetamide respectively, followed by a nucleophilic addition of the amino/imino group to the double bond, afforded 1,2,5,6-tetrahydro-6-(2-imino- $2 \mathrm{H}$ chromen-3-yl)-2-oxo(thioxo)-4-phenyl-6-(phenylamino) pyridine-3-carbonitriles 29a - 29e , respectively (Scheme 8). The IR spectrum of 29a showed the presence of absorption bands at $v 1689$ - 1705, 2220 and 3188 - 3244 $\mathrm{cm}^{-1}$ due to $\mathrm{C}=\mathrm{O}, \mathrm{CN}$, and $\mathrm{NH}$ functions, respectively, and the ${ }^{1} \mathrm{H}$ NMR spectrum (DMSO) displayed three singlet signals at $\delta 2.9,4.1$ and 8.1 due to the proton at C-5 of pyridine, $\mathrm{NH}$-amine and $\mathrm{NH}$ amide, respectively, and a multiplet at $7.2-7.6 \mathrm{ppm}$, respectively. The MS of 29a showed a peak at $m / z 432\left([\mathrm{M}]^{+}, 65 \%\right)$.

Similarly, compound 24a reacted with 2-chloroacetamide to afford 3-chloro-5, 6-dihydro-6-(2-imino-2H-chromen-3-yl)-4-phenyl-6-(phenylamino)pyridin-2(1H)-one 30, (Scheme 9). Also, 2-(cyanomethyl) benzimidazole, reacted with the chalcone $24 a$, in ethanol using piperidine as a catalyst gave the 2-(2-imino- $2 H$-chromen-3-yl)-4phenyl-2-(phenylamino) pyrido[1,2-a]ben-zimidazolo-5carbonitriles, derivative probably via initial condensation of the activated methylene group with the carbonyl function releasing water, followed by a nucleophilic addition of the NH group at the double bond of compound 24a to afford 31, (Scheme 9). The structure of 31 was assigned based on IR, ${ }^{1} \mathrm{H}$ NMR, and mass spectra. The IR spectrum showed the presence of absorption bands at $v 2220 \mathrm{~cm}^{-1}$ and $3188-3244 \mathrm{~cm}^{-1}$ due to $\mathrm{CN}$ and $\mathrm{NH}$ functions respectively, while that due to $\mathrm{C}=\mathrm{O}$ was completely disappeared, and the ${ }^{1} \mathrm{H}$ NMR spectrum $\left(\mathrm{CDCl}_{3}\right)$ displayed three singlet at $\delta 2.9,4.1$ and 11.5 due to the $\mathrm{C}-3$ proton, $\mathrm{NH}$-amine and $\mathrm{NH}$-imino protons respectively, and a multiplet at $\delta 6.9-7.6 \mathrm{ppm}$, for aromatic protons. The MS showed the $[\mathrm{M}]^{+}$ion at $m / z 505(35 \%)$. Similarly, the chalcone 24a was reacted with amino phenols in ethanolic solution containing a catalytic amount of piperidine to give 2,3-dihydro-2-(2-imino- $2 H$-chromen-3-yl)- $N, 4$-diphenyl-benzo $[b][1,4]$ azepine derivatives , 32a, b (Scheme 9). The IR spectrum of 32a showed the presence of absorption bands at $v 3289 \mathrm{~cm}^{-1}$ due to $\mathrm{NH}$ function, the ${ }^{1} \mathrm{H}$ NMR spectrum (DMSO) of 32a displayed three singlet signals at $\delta 2.9,4.1$ and 11.5 due to the $\mathrm{C}-3$ protons, $\mathrm{NH}-$ amine and $\mathrm{NH}$-imino protons respectively, and a multiplet at $6.7-7.3 \mathrm{ppm}$ for aromatic protons. While, the MS of 32a showed a peak at $m / z 457\left([\mathrm{M}]^{+}, 55 \%\right)$. Also, comund 24a was reacted with 2-aminoprop-1-ene-1, 1,3-tricarbonitrile and/or ethyl 3-amino-2,4-dicyanobut-2-enoate to afford 2-(dicyano- methylene)-1,2,5,6-tetrahydro-6-(2imino-2H-chromen-3-yl)-4-phenyl-6-(phenylamino)pyridine-3-carbonitrile, 33a and 2-(1-cyano-2-oxobutylidene)1,2,5,6-tetrahydro-6-(2-imino-2 $H$-chromen-3-yl)-4-phenyl6-(phenylamino) pyridine-3-carbonitrile 33b, respectively. The structure of these products was established based on the IR spectrum of 33a which showed the presence of absorption bands at $v 2219$ and $3289 \mathrm{~cm}^{-1}$ due to $\mathrm{C}=\mathrm{O}$ and $\mathrm{NH}$ functions, respectively. The ${ }^{1} \mathrm{H}$ NMR spectrum (DMSO) of 33a displayed two singlet signals at $\delta 2.9$ and 4.1 due to the $\mathrm{C}-3$ protons and $\mathrm{NH}$-amine and a multiplet at $6.7-7.3$ ppm for aromatic protons. While, the MS of 33a showed a peak at $m / z 482\left([\mathrm{M}+2]^{+}, 35 \%\right)$.

On the other hand, the new synthesized Schiff's bases 2-imino- $N$, $N$ '-diphenyl- $2 H$-chromene-3-carboxamidines, 25a - 25e, were used to prepare new compounds. Thus, cycloaddition of chloroacetyl chloride to 25a - 25e proceeded smoothly in ethanolic solution containing triethylamine as a catalyst to give the $\beta$-lactam, derivatives 34a 34e, (Scheme 10). The IR spectra of 34a - 34e showed the

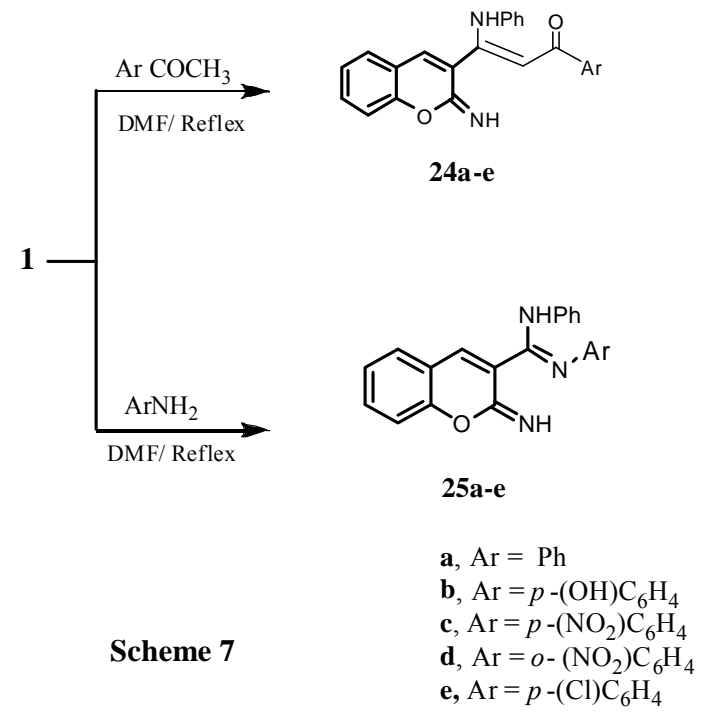

Scheme 7. Synthetic rpute to chalcones 24a - e and Schiff's bases 25a - e. 


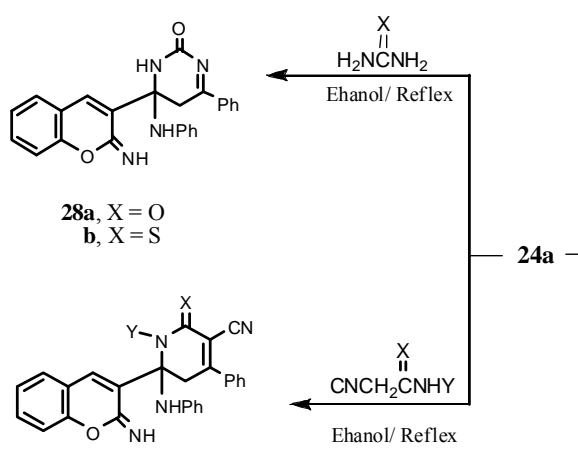

$\begin{aligned} \text { 29a, } \mathrm{X} & =\mathrm{O}, \mathrm{Y}=\mathrm{H} \\ \text { b, } \mathrm{X} & =\mathrm{S}, \mathrm{Y}=\mathrm{H} \\ \text { c, } \mathrm{X} & =\mathrm{O}, \mathrm{Y}=p-\left(\mathrm{CH}_{3}\right) \mathrm{C}_{6} \mathrm{H}_{4} \\ \text { d, } \mathrm{X} & =\mathrm{O}, \mathrm{Y}=\mathrm{NH}_{2} \\ \text { e, } \mathrm{X} & =\mathrm{O}, \mathrm{Y}=\mathrm{Ph}\end{aligned}$

Scheme 8

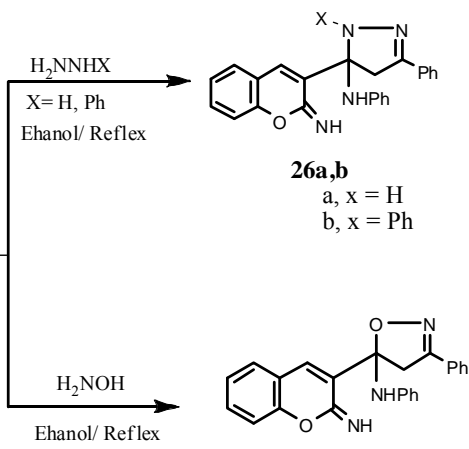

27

Scheme 8. Synthetic rpute to isolated pyrazole, isoxazole, pyrimidine and pyridine derivatives 26ab, 27, 28a,b and 29a - e.

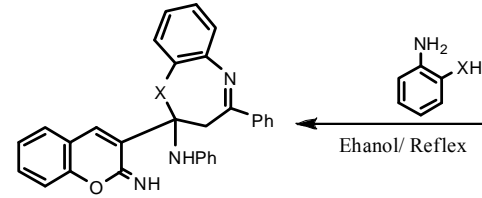

$\begin{aligned} 32 a, X & =O \\ \text { b, } X & =S\end{aligned}$

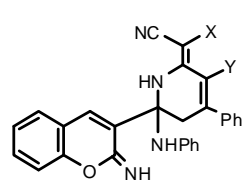

33a, $\mathrm{Y}=\mathrm{X}=\mathrm{CN}$

b, $\mathrm{Y}=\mathrm{CN}, \mathrm{X}=\mathrm{CO}_{2} \mathrm{C}_{2} \mathrm{H}_{5}$

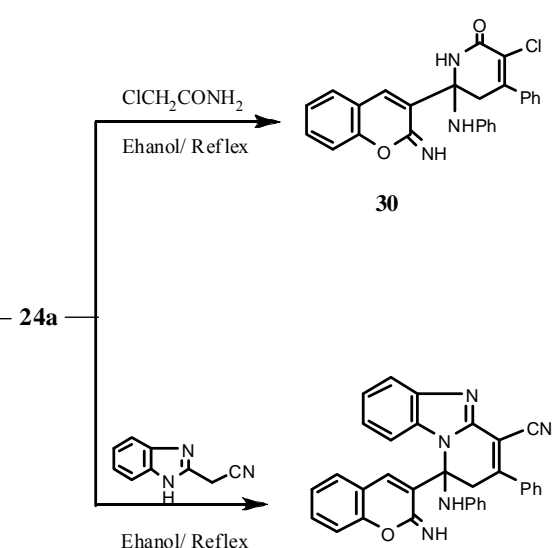

31

Scheme 9. Synthetic rpute for the preparation of compounds 30 - 33a,b.

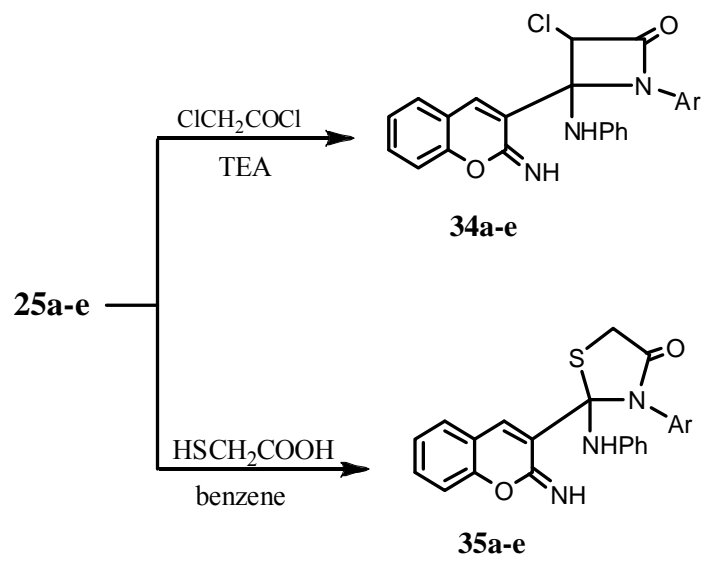

Scheme 10
a, $\mathrm{Ar}=\mathrm{Ph}$
b, $\mathrm{Ar}=p-(\mathrm{OH}) \mathrm{C}_{6} \mathrm{H}_{4}$
c, $\mathrm{Ar}=p-\left(\mathrm{NO}_{2}\right) \mathrm{C}_{6} \mathrm{H}_{4}$
d, $\mathrm{Ar}=o-\left(\mathrm{NO}_{2}\right) \mathrm{C}_{6} \mathrm{H}_{4}$
e, $\mathrm{Ar}=p-(\mathrm{Cl}) \mathrm{C}_{6} \mathrm{H}_{4}$

Scheme 10. Synthetic rpute for the preparation of $\beta$-latame 34a,b. and thiazolodinone derivatives $35 a$ - e. 
presence of absorption bands due to $\mathrm{C}=\mathrm{O}$ and $\mathrm{NH}$ functions respectively. The ${ }^{1} \mathrm{H}$ NMR spectrum (DMSO) of 34c showed three singlet signals at $\delta 4.1,5.2$ and $11.5 \mathrm{ppm}$ for $\mathrm{NH}$-amine, the proton at $\mathrm{C} 3$ of the $\beta$-lactam unit and $\mathrm{NH}$-imino protons respectively and a multiplet at $\delta 7.1$ 7.6 for aromatic protons. The MS of $34 \mathrm{c}$ showed the $[\mathrm{M}]^{+}$ ion at $\mathrm{m} / \mathrm{z} 460$ (40\%). Also, the Schiff's bases 25a - 25e were reacted with equimolar ratio of thioglycolic acid in boiling benzene using water separator system, the thiol group of thioglycolic acid could be added to the imino carbon atoms of Schiff's bases 25a - 25e followed by smooth cyclization to afford 2-(2-imino- $2 H$-chromen-3yl)-3-phenyl-2-(phenylamino) thiazolidin-4-ones, 35a 35e (Scheme 10). The IR spectra of 35 showed bands due to $\mathrm{CS}, \mathrm{CO}$ and NH groups, respectively. While, the ${ }^{1} \mathrm{HNMR}$ spectrum of 35a (DMSO) showed three singlet signals at $\delta$ $3.8,4.1$ and 5.8 for the methylene of thiazolidinone, $\mathrm{NH}-$ amine and $\mathrm{NH}$-imino protons respectively and a multiplet at $\delta 6.8-7.8$ for aromatic protons. In the MS of 35a showed $\mathrm{m} / \mathrm{z}$ at $411\left([\mathrm{M}-2]^{+}, 35 \%\right)$.

\section{Conclusion}

Despite the several existing methods for the synthesis of chromene derivatives, there still is demand for general strategies, which can efficiently provide variously substituted chromene systems. Thus, this work opened a new avenue for the synthesis of a variety of 2-imino- $N$-phenyl-2H-chromene-3-carboxamide, derivatives.

\section{REFERENCES}

[1] A. M. El-Agrody M. S. Abd El-Latif, N. A. El-Hady, A. H. Fakery and A. H. Bedair, "Heteroaromatization with 4-Hydroxycoumarin Part II: Synthesis of Some New Pyrano[2, 3-d]pyrimidines, $[1,2,4]$ triazolo[1,5-c]pyrimidines and Pyrimido[1,6-b]-[1,2,4]triazine Derivatives," Molecules, Vol. 6, No. 6, 2001, pp. 519-527. doi:10.3390/60600519

[2] K. Mazaahir, S. Shilpi, M. R. K. Khalilur and S. T. Sharanjit, "Aqua Mediated Synthesis of Substituted 2-Amino4H-chromenes and in Vitro Study as Antibacterial Agents," Bioorganic \& Medicinal Chemistry Letters, Vol. 15, No. 19, 2005, pp. 4295-4298. doi:10.1016/j.bmcl.2005.06.041

[3] A. Luke, P. Soizic, S. Brigitte, M. Sylvie, K. Michel, T. C. Stewart, T. François and L. J. Yves, European Journal of Medicinal Chemistry, 2009, pp. 1-9.

[4] B. Andrea, V. Nicole, T. Jakob, H. Sonja, B. Stefan and E. M. Christa, "Synthesis and Pharmacological Evaluation of Coumarin Derivatives as Cannabinoid Receptor Antagonists and Inverse Agonists," Bioorganic \& Medicinal Chemistry, Vol. 17, No. 7, 2009, pp. 2842-2851. doi:10.1016/j.bmc.2009.02.027

[5] P. Vassiliki, K. K. Ioannis, M. Panagiotis, P. Nicole and A. Ioanna, "Synthesis and Free Radical Scavenging Activity of Some New Spiropyranocoumarins," Bioorganic \& Medicinal Chemistry Letters, Vol. 18, No. 21, 2008, pp. 57815784. doi:10.1016/j.bmcl.2008.09.065
[6] N. Tadigoppula, K. Tanvir, N. Shweta, G. Neena and G. Suman, Bioorganic \& Medicinal Chemistry, Vol. 13, No. 13, 2005, pp. 6543-6550.

[7] K. William, K. Shailaja, S. C. Jiang, H. Zhang, J. H. Zhao, S. J. Jia, L. F. Xu, C. Crogan-Grundya, R. Denis, N. Barriault, L. Vaillancourt, S. Charron, J. Dodd, G. Attardo, D. Labrecque, S. Lamothe, H. Gourdeau, B. Tseng, J. Drewea and S. X. Cai, "Discovery of 4-Aryl-4H-chromenes as a New Series of Apoptosis Inducers Using a Cell- and Caspase-Based High-Throughput Screening Assay. 2. Structure-Activity Relationships of the 7- and 5-, 6-, 8-Positions," Bioorganic \& Medicinal Chemistry Letters, Vol. 15, No. 21, 2005, pp. 4745-4751. doi:10.1016/j.bmcl.2005.07.066

[8] G. Danielle, V. Samuel, L. M. Vijay, C. Frédéric, V. Guillaume, M. Florence, J. Philippe and G. René, "The Synthesis of New, Selected Analogues of the Pro-Apoptotic and Anticancer Molecule HA 14-1," Tetrahedron Letters, Vol. 49, No. 20, 2008, pp. 3276-3278. doi:10.1016/j.tetlet.2008.03.070

[9] T. El-Sayed Ali, S. A.-A. Abdel-Aziz, H. M. El-Shaaer, F. I. Hanafy and A. Z. El-Fauomy, Turkish Journal of Chemistry, Vol. 32, 2008, pp. 365-374.

[10] M. K. Mostafa, H. F. A. Ashraf, A. E. Fathy and M. E. Ahmed, IL Farmaco, Vol. 57, 2002, pp. 715-722.

[11] A. G. Martinez and L. J. Marco, "Friedländer Reaction on 2-Amino-3-cyano-4H-pyrans: Synthesis of Derivatives of 4H-Pyran [2,3-b] Quinoline, New Tacrine Analogues," Bioorganic \& Medicinal Chemistry Letters, Vol. 7, No. 24, 1997, pp. $3165-3170$. doi:10.1016/S0960-894X(97)10165-2

[12] C. P. Dell and C. W. Smith, European Patent No. EP537949, 1993.

[13] R. L. Dorta, A. Martin, E. Suárez and C. Betancor, "Endipitous Acid-Catalyzed Rearrangement of 13-Methoxy1,6,8-trioxadispiro[4.1.5.3]pentadecane to 3-Chroman-5ylpropan-1-ol," The Journal of Organic Chemistry, Vol. 62, No. 7, 1997, pp. 2273-2274. doi:10.1021/j0962065j

[14] Y. S. Vyacheslav, S. M. Vladimir and A. I. Roman, "Reactions of 3-(Polyfluoroacyl)chromones with Hydroxylamine. The First Synthesis of 3-Cyano-2-(polyfluoroalkyl)chromones," Tetrahedron Letters, Vol. 47, No. 48, 2006, pp. 8543-8546. doi:10.1016/j.tetlet.2006.09.136

[15] D. Brian, Z. Liren, T. Junko, P. Joseph, H. Sarah, C. Brett, E. H. Christopher, W. Jenny, N. Christi, M. Ajay, S. David, W. Warren and S. G. Val, Bioorganic \& Medicinal Chemistry Letters, Vol. 16, 2006, pp. 4237-4242.

[16] Y.-W. Guo, Y.-L. Shi, H.-B. Li and M. Shi, "Reactions of Salicyl N-Tosylimines or Salicylaldehydes with Diethyl Acetylenedicarboxylate for the Synthesis of Highly Functionalized Chromenes," Tetrahedron, Vol. 62, No. 25, 2006, pp. 5875-5882. doi:10.1016/j.tet.2006.04.011

[17] Y. Seiji, M. Mikiko, M. Yohei, M. Masahiro and H. Yoshiro, Tetrahedron Letters, Vol. 45, 2004, pp. 6971-6973.

[18] E. H. E. Galal and H. H. E. Ahmed, "Activated Nitriles in Heterocyclic Synthesis. Novel Synthesis of 5-Imino-5H[1]benzopyrano[3,4-c]pyridine-4(3H)-thiones and Their Oxo Analogues," Bulletin of the Chemical Society of Japan, Vol. 63, No. 4, 1990, pp. 1230-1232. 
doi: $10.1246 /$ bcsj.63.1230

[19] M. M. Rafat, Z. S. Hoda and H. E. Mohamed, Gazzetta Chimica Italiana, 1992, 122.

[20] M. K. Sergiy, E. B. Igor, M. S. Konstantyn, P. C. Valentyn and V. B. Yaroslav, "A New Pathway to 3-Hetaryl-2-oxo2H-chromenes: On the Proposed Mechanisms for the Reaction of 3-Carbamoyl-2-iminochromenes with Dinu- cleophiles," Molecules, Vol. 5, No. 10, 2000, pp. 11461165. doi: $10.3390 / 51001146$

[21] S. Andrea, V. Daniel, L. André and F. L'ubomír, ARKIVOC, Vol. 6, 2001, pp. 122-128.

[22] T. Steinfuhrer, A. Hantschmann, M. Pietsch and M. Weissenfels, Liebigs Ann. Chem., Vol. 23, 1992. 